TRANSACTIONS OF THE

AMERICAN MATHEMATICAL SOCIETY

Volume 356, Number 7 , Pages 2963-2988

S 0002-9947(04)03434-8

Article electronically published on February 27, 2004

\title{
HERMITIAN METRICS INDUCING THE POINCARÉ METRIC, IN THE LEAVES OF A SINGULAR HOLOMORPHIC FOLIATION BY CURVES
}

\author{
A. LINS NETO AND J. C. CANILLE MARTINS
}

\begin{abstract}
In this paper we consider the problem of uniformization of the leaves of a holomorphic foliation by curves in a complex manifold $M$. We consider the following problems: 1 . When is the uniformization function $\lambda_{g}$, with respect to some metric $g$, continuous? It is known that the metric $\frac{g}{4 \lambda_{g}}$ induces the Poincaré metric on the leaves. 2. When is the metric $\frac{g}{4 \lambda_{g}}$ complete? We extend the concept of ultra-hyperbolic metric, introduced by Ahlfors in 1938, for singular foliations by curves, and we prove that if there exists a complete ultra-hyperbolic metric $g$, then $\lambda_{g}$ is continuous and $\frac{g}{4 \lambda_{g}}$ is complete. In some local cases we construct such metrics, including the saddle-node (Theorem 1) and singularities given by vector fields with the first non-zero jet isolated (Theorem 2). We also give an example where for any metric $g, \frac{g}{4 \lambda_{g}}$ is not complete $(\S 3.2)$.
\end{abstract}

\section{$\S 1$. INTRODUCTION}

We will consider the following situation: let $\mathcal{F}$ be a holomorphic foliation by curves in a complex manifold $M$. We will denote by $\operatorname{sing}(\mathcal{F})$ the singular set of $\mathcal{F}$ and by $L_{p}$ the leaf of $\mathcal{F}$ through a point $p \in M \backslash \operatorname{sing}(\mathcal{F}):=V$. Let

$$
\mathcal{H}(\mathbb{D}, M)=\{f: \mathbb{D} \rightarrow M ; f \text { is holomorphic }\},
$$

where $\mathbb{D}$ is the Poincaré disc $\{z \in \mathbb{C} ;|z|<1\}$, and let

$$
\mathcal{H}(\mathbb{D}, \mathcal{F})=\{f \in \mathcal{H}(\mathbb{D}, V) ; f(\mathbb{D}) \subset L, \text { where } L \text { is a leaf of } \mathcal{F}\} .
$$

Given a hermitian metric $g$ in $M$, define $\lambda_{\mathcal{F}, g}=\lambda: V \rightarrow(0,+\infty]$ by

$$
\lambda(p)=\sup \left\{\left|\alpha^{\prime}(0)\right|_{g}^{2} ; \alpha \in \mathcal{H}(\mathbb{D}, \mathcal{F}) \text { and } \alpha(0)=p\right\},
$$

where $\left|v_{p}\right|_{g}=\sqrt{g_{p}\left(v_{p}, v_{p}\right)}$ denotes the norm of a vector $v_{p} \in T_{p} M$.

The following facts are well known:

(I) $\lambda(p)<+\infty$ if, and only if, $L_{p}$ is hyperbolic, that is, its holomorphic universal covering is the Poincaré disc. In this case we have $\lambda(p)=\left|\alpha^{\prime}(0)\right|_{g}^{2}$, where $\alpha: \mathbb{D} \rightarrow L_{p}$ is an uniformization of $L_{p}$ such that $\alpha(0)=p$ (cf. [V]).

(II) If $L_{p}$ is hyperbolic and $\alpha: \mathbb{D} \rightarrow L_{p}$ is a uniformization such that $\alpha(0)=p$, then

$$
\lambda(\alpha(z))=\left(1-|z|^{2}\right)^{2}\left|\alpha^{\prime}(z)\right|_{g}^{2} .
$$

Received by the editors June 19, 2002 and, in revised form, June 2, 2003.

2000 Mathematics Subject Classification. Primary 37F75.

This work was supported by FAPESP. 
In particular, $\left.\lambda\right|_{L_{p}}$ is continuous and $\frac{4 g}{\lambda}$ induces the Poincaré metric on $L_{p}$ (cf. [P], [V], [LN] and [C-G]).

(III) $\lambda$ is lower semicontinuous (cf. [C], C-G] and [V]).

In the case where all leaves of $\mathcal{F}$ are hyperbolic we will use the following notation:

$$
\mathcal{U}=\{\alpha \in \mathcal{H}(\mathbb{D}, \mathcal{F}) ; \alpha \text { is an uniformization of some leaf of } \mathcal{F}\} .
$$

Now, let $\mu$ be a hermitian metric on $M \backslash \operatorname{sing}(\mathcal{F})$ of class $C^{r}, r \geq 2$. Define $k_{\mu}: M \backslash \operatorname{sing}(\mathcal{F}) \rightarrow \mathbb{R}$ by

$$
k_{\mu}(p)=\text { Gaussian curvature of } L_{p} \text { at } p \text { in the metric }\left.\mu\right|_{L_{p}} .
$$

The following result is known (cf. [LN]):

Theorem A. Let $M, \mathcal{F}$ and $\operatorname{sing}(\mathcal{F})$ be as before. Suppose that there exists a hermitian metric $\mu$ of class $C^{r}(r \geq 2)$ on $M \backslash \operatorname{sing}(\mathcal{F})$, such that:

(a) $k_{\mu} \leq-a^{2}, a>0$.

Then all leaves of $\mathcal{F}$ are hyperbolic. Furthermore, if

(b) $\mu$ is complete (that is, the distance induced by $\mu$ on $M \backslash \operatorname{sing}(\mathcal{F})$ is complete).

(c) $M$ is compact and all connected components of $\operatorname{sing}(\mathcal{F})$ are hyperbolic in the sense of $[\mathrm{K}]$.

Then $\mathcal{U}$ is relatively compact in the topology of uniform convergence in the compact parts of $\mathbb{D}$. Moreover, if $\operatorname{sing}(\mathcal{F})$ is finite, then $\overline{\mathcal{U}}=\mathcal{U} \cup S$, where

$$
S=\{\alpha: \mathbb{D} \rightarrow \operatorname{sing}(\mathcal{F}) ; \alpha \text { is a constant }\} .
$$

Remark 1. If $M$ is not compact, then Theorem A can be stated as follows:

Theorem A.1. Let $M, \mathcal{F}$ and $\operatorname{sing}(\mathcal{F})$ be as in Theorem A. Suppose that there exists a hermitian metric $\mu$ of class $C^{r}(r \geq 2)$ on $M \backslash \operatorname{sing}(\mathcal{F})$, satisfying (a), (b) of Theorem A and

(c.1) $\operatorname{sing}(\mathcal{F})$ is discrete.

Then, any sequence $\left(\alpha_{n}\right)_{n \geq 1}$ in $\mathcal{U}$, such that the sequence $\left(\alpha_{n}(0)\right)_{n \geq 1}$ is convergent, is normal. Furthermore, we have that:

(1) If $\lim _{n \rightarrow \infty} \alpha_{n}(0)=p \in \operatorname{sing}(\mathcal{F})$, then $\left(\alpha_{n}\right)_{n \geq 1}$ converges to the constant $p$.

(2) If $\lim _{n \rightarrow \infty} \alpha_{n}(0)=p \notin \operatorname{sing}(\mathcal{F})$, then any convergent subsequence of $\left(\alpha_{n}\right)_{n \geq 1}$ converges to an uniformization of $L_{p}$. Moreover, the sequence converges if, and only if, $\left(\alpha_{n}^{\prime}(0)\right)_{n \geq 1}$ is convergent.

The proof of the above result is analogous to the proof of Theorem A found in LN]. In the next section we will see a generalization of Theorem A.1, in which we will introduce the concept of " $\mathcal{F}$-ultrahyperbolic metric", due to Ahlfors, in the case of Riemann surfaces. Moreover, we will see that in this case the function $\lambda=\lambda_{\mathcal{F}, g}$, defined in $(*)$, is continuous and that the metric $\frac{4 g}{\lambda}$ is complete. In fact, in this paper we will be mainly concerned with the following problems.

Problem 1. Let $M$ and $\mathcal{F}$ be as before and let $g$ be a $C^{r}(r \geq 2)$ hermitian metric in $M$. Suppose that all leaves of $\mathcal{F}$ are hyperbolic and let $\lambda=\lambda_{\mathcal{F}, g}$ be as in (*). Under which conditions on $M$ and $\mathcal{F}$ is $\lambda$ continuous?

Problem 2. Let $M, \mathcal{F}, g$ and $\lambda$ be as in Problem 1 and suppose that $\lambda$ is continuous. Under which conditions on $M, \mathcal{F}$ and $g$ is the metric $\frac{4 g}{\lambda}$ complete?

So, for instance, if the hypotheses of Theorem A.1 are fullfilled, then $\lambda=\lambda_{\mathcal{F}, \mu}$ is continuous and the metric $\frac{4 \mu}{\lambda}$ is complete. 
In $\S 3$ we will study Problems 1 and 2 in the local case. Before stating our results in this direction, we will give a definition. We will consider the following situation:

Let $X$ be a germ of holomorphic vector field at $0 \in \mathbb{C}^{n}$, with an isolated singularity at 0 and $\mathcal{F}$ the germ of foliation by curves defined by $X$. Given a bounded neighborhood $U$ of 0 such that there exists a representative of $X$ defined in $U$, then all leaves of $\left.\mathcal{F}\right|_{U}$ are hyperbolic (from Liouville's Theorem, all holomorphic functions $f: \mathbb{C} \rightarrow U$ are constant). In this case, if $g$ is a hermitian metric on $U$, we can define $\lambda_{\mathcal{F}, g}$ as in $(*)$.

Definition 1. Let $X, \mathcal{F}$ be as above, and let $g$ be a hermitian metric defined in a neighborhood of $0 \in \mathbb{C}^{n}$. Given a neighborhood $U$ of 0 such that there exists a representative of $X$ and $g$ defined in $U$, let $\mathcal{F}_{U}$ be the restriction of $\mathcal{F}$ to $U$ and $\lambda_{U}=\lambda_{\mathcal{F}_{U}, g}$. We will say that $X$ or $\mathcal{F}$ satisfies property $\mathbf{P . 1}$ with respect to $g$, if there exists a neighborhood $U$ of 0 as above, such that $\lambda_{U}$ is continuous on $U \backslash\{0\}$.

Let $h$ be a hermitian metric on $W \backslash\{0\}$, where $W$ is a neighborhood of $0 \in \mathbb{C}^{n}$. We say that $h$ is complete at 0 , if for any $C^{1}$ path $\gamma:[0,1] \rightarrow U$ such that $\gamma(0)=0$ and $\gamma(t) \neq 0$ for $t \neq 0$, then the length of $\gamma$ with respect to $h$ is $+\infty$, that is,

$$
\ell(\gamma)=\int_{\gamma} \sqrt{h}=+\infty
$$

Let $g$ be a hermitian metric on a neighborhood $U$ of 0 and $\mu_{U}=\frac{\left.4 g\right|_{U}}{\lambda_{U}}$. We say that $X$ or $\mathcal{F}$ satisfies property $\mathbf{P . 2}$ with respect to $g$, if there exists a neighborhood $U$ of 0 such that $\mu_{U}$ is complete at 0 .

Remark 2. In most cases we will consider $g=|d z|^{2}=\sum_{j=1}^{n}\left|d z_{j}\right|^{2}$ the euclidean metric on $\mathbb{C}^{n}$. It is not difficult see that, if $\mathcal{F}$ satisfies property $\mathbf{P . 1}$ or $\mathbf{P . 2}$, with respect to $|d z|^{2}$, then it satisfies property $\mathbf{P . 1}$, or $\mathbf{P . 2}$, with respect to any other hermitian metric. Due to this fact, we will say that $\mathcal{F}$ satisfies property $\mathbf{P . 1}$ (resp. P.2), if it satisfies property $\mathbf{P . 1}$ (resp. P.2) with respect to some metric.

Remark 3. It follows from the Corollary of Proposition 4 of $\S 2.3$ that if the germ of $\mathcal{F}$ satisfies property $\mathbf{P . 2}$, then it satisfies property $\mathbf{P . 1 .}$

In the direction of Problems 1 and 2 (in the local case), the following result is known (cf. [LN]):

Proposition B. If $X$ has a nondegenerated singularity at $0 \in \mathbb{C}^{n}$, then $X$ satisfies properties $\mathbf{P . 1}$ and $\mathbf{P . 2}$.

In $\S 2$ we will introduce the notion of " $\mathcal{F}$-ultrahyperbolic" metric (cf. [Ah-2]). This notion, togheter with a lemma due to Ahlfors, will allow us to generalize the above result for continuous metrics. We will apply this generalization in some results which will be proved in $\S 3$. Our main result in $\S 3$ will be the following:

Theorem 1. Let $X$ be a holomorphic vector field with a saddle-node at $0 \in \mathbb{C}^{2}$. Then $X$ satisfies properties P.1 and P.2.

Recall that $p$ is a saddle node for $X$, if $D X(p)$ has eigenvalues $\lambda_{1} \neq 0$ and $\lambda_{2}=0$. Another result that we will prove in $\S 3$ is the following:

Theorem 2. Let $X$ be a holomorphic vector field with a singularity at $0 \in \mathbb{C}^{n}$ and Taylor series at 0 of the type $X=X_{k}+$ h.o.t., where the components of $X_{k}$ are homogeneous polynomials of degree $k$ and h.o.t. means "highter order terms". 
Suppose that 0 is an isolated singularity of $X_{k}$. Then $X$ satisfies properties $\mathbf{P . 1}$ and $\mathbf{P . 2}$.

In $\S 3$ we will also see that the foliation in $\mathbb{C}^{2}$, whose leaves are the level surfaces of $y^{2}-x^{3}$, given by the vector field $X=2 y \partial / \partial x+3 x^{2} \partial / \partial y$, does not satisfy property P.2 at 0 .

Another problem that we will consider is the following:

Problem 3. Find the growth order of $\lambda_{\mathcal{F}, g}(z)=\lambda(z)$, or of $\frac{\left.4 g\right|_{U}}{\lambda_{\mathcal{F}, g}}$, near an isolated singular point $p$ of $\mathcal{F}$, when $z$ goes to $p$.

In this direction we will prove the following result:

Theorem 3. Let $X$ be a holomorphic vector field in an open bounded ball $B=$ $B(0, r)=\left\{z \in \mathbb{C}^{n} ;|z|<r\right\} \subset \mathbb{C}^{n}$ with a singularity at $0 \in B$ and let $\mathcal{F}$ be the foliation defined by $X$. Suppose that $X=X_{k}+$ h.o.t. is as in Theorem 2. Let $g$ be a $C^{m}, m \geq 0$, hermitian metric in $B, \lambda=\lambda_{\mathcal{F}, g}$ and $\mu=\frac{4 g}{\lambda}$. We have the following:

(a) If $k=1$, that is, 0 is a nondegenerated singularity, then there exist $K>1$ and $0<\rho<r$ such that

$$
K^{-1} \frac{|d z|^{2}}{|z|^{2}(\ln |z|)^{2}} \leq \mu \leq K \frac{|d z|^{2}}{|z|^{2}(\ln |z|)^{2}}, \forall z \in B(0, \rho) .
$$

(b) If $k>1$, then there exist $K>1$ and $0<\rho<r$ such that

$$
K^{-1} \frac{|d z|^{2}}{|z|^{2}(\ln |z|)^{2}} \leq \mu \leq K \frac{|d z|^{2}}{|z|^{2}}, \forall z \in B(0, \rho) .
$$

Remark 4. It is possible to prove that the inequalities in (b) are the best possible in the general case (involving expressions which depend only on $|z|$ ). In $\S 3$ we will sketch the proof of this fact (Example 2).

We would like to propose the following questions:

Question 1. Does every isolated singularity satisfy property (P.1)?

For the next question, let $\mathcal{F}$ be a holomorphic singular foliation in a compact manifold $M$ of complex dimension $n \geq 2$. Suppose that $\operatorname{sing}(\mathcal{F})$ is finite and that all leaves of $\mathcal{F}$ are hyperbolic.

Question 2. Is $\mathcal{H}(\mathbb{D}, \mathcal{F})$ normal?

Question 3. Let $g$ be a hermitian metric in $M, \lambda=\lambda_{\mathcal{F}, g}$ and $\mu=\frac{4 g}{\lambda}$. Are $\lambda$ and $\mu$ continuous?

\section{§2. Preliminary Results}

In this section we state some results which will be used in the proofs of the theorems stated in $\S 1$.

$\S 2.1$. The generalized Ahlfors lemma. In 1938 Ahlfors introduced the concept of an ultrahyperbolic conformal metric in a Riemann surface (cf. [Ah-1] and [Ah-2]). A conformal pseudo-riemannian metric in a Riemann surface $S$ is a quadratic form $g$ in $S$, which can be written in a holomorphic coordinate system $(z, U)$ as $g_{z}=$ $f(z)|d z|^{2}$, where $f$ is a continuous function on the open set $z(U) \subset \mathbb{C}$, such that 
$f \geq 0$ and the set $f^{-1}(0)$ is discrete. In this case, we define the length of a $C^{1}$ curve $\gamma:[a, b] \rightarrow S$ with respect to $g$ by

$$
\ell_{g}(\gamma)=\int_{\gamma} \sqrt{g}=\int_{a}^{b} \sqrt{\left|\gamma^{\prime}(t)\right|_{g}^{2}} d t
$$

It is not difficult to see that if $\gamma(a) \neq \gamma(b)$, then $\ell(\gamma)>0$. Therefore we can define a distance in $S$ by

$$
d_{g}(p, q)=\inf \left\{\ell_{g}(\gamma) ; \gamma \text { is a } C^{1} \text { curve joining } p \text { and } q\right\} .
$$

Definition 4. Let $g$ be a conformal pseudo-Riemannian metric in $S$. We say that $g$ is ultrahyperbolic of curvature bounded by $a<0$ if for every $p \in U$ with $g_{p} \neq 0$, there exist a holomorphic coordinate system $(z, U)$ around $p$ with $z(p)=0$, $\left.g\right|_{U}=f(z)|d z|^{2}, f>0$, in $z(U)=V$, and a positive $C^{2}$ function $h$ in $V$, such that $h(0)=f(0), h \leq f$, in $V$ and the gaussian curvature of the metric $h(z)|d z|^{2}$, say $k$, satisfies $k \leq a<0$ in $V$. We say that $g$ is ultrahyperbolic, if it is ultrahyperbolic of curvature bounded by $a<0$, for some $a<0$.

Remark 5. We would like to observe that Ahlfors' definition is more general, in the sense that he demands that $f$ is just upper semicontinuous. However, in this paper all metrics that will appear will be continuous.

Remark 6. It is not difficult to see that this concept is well defined and invariant by biholomorphisms. Moreover, if $S_{1}$ and $S_{2}$ are two Riemann surfaces, $F: S_{1} \rightarrow S_{2}$ is a holomorphic nonconstant map and $g$ is a conformal pseudo-Riemannian metric in $S_{2}$, ultrahyperbolic of curvature bounded by $a<0$, then $F^{*}(g)$ is also.

The following result was proved by Ahlfors:

Theorem (Ahlfors Lemma). Let $S$ be a Riemann surface and suppose that there is a conformal pseudo-Riemannian metric $g$ in $S$ which is ultrahyperbolic of curvature bounded by $-a^{2}<0$. Then $S$ is hyperbolic and $g \leq \frac{1}{a^{2}} \mathcal{P}_{S}$, where $\mathcal{P}_{S}$ is the Poincaré metric of $S$. In particular, we have

$$
d_{g}(p, q) \leq \frac{1}{a} d_{\mathcal{P}}(p, q), \forall p, q \in S,
$$

where $d_{\mathcal{P}}$ is the Poincaré distance in $S$.

Let us state anoother result that will be used.

Proposition 1. Let $S$ be a Riemann surface and let $g_{1}, \ldots, g_{k}$ be ultrahyperbolic on $S$ of curvature bounded by $-a_{1}<0, \ldots,-a_{k}<0$, respectively. Define $g_{+}=$ $g_{1}+\ldots+g_{k}$ and $g_{M}=\max \left\{g_{1}, \ldots, g_{k}\right\}$. Then $g_{+}$and $g_{M}$ are ultrahyperbolic of curvature bounded by $-a_{+}<0$ and $-a_{M}<0$, where $\frac{1}{a_{+}}=\frac{1}{a_{1}}+\ldots+\frac{1}{a_{k}}$ and $a_{M}=\min \left\{a_{1}, \ldots, a_{k}\right\}$, respectively.

Proof. We observe that if $g_{j}=f_{j}(z)|d z|^{2}$, locally, then

$$
\max \left\{g_{1}, \ldots, g_{k}\right\}=\max \left\{f_{1}, \ldots, f_{k}\right\}|d z|^{2} .
$$

Therefore, in the case of $g_{M}$ the proof follows directly from Definition 4. The case of $g_{+}$follows from $[\mathrm{K}]$ (chapter I), in the case where $g_{1}, \ldots, g_{k}$ are positive and of class $C^{2}$. In the general case, the proof follows from this case and the definition. 
$\S 2.2$. Foliated Ahlfors lemma. We now introduce the notion of an $\mathcal{F}$-ultrahyperbolic metric for a foliation $\mathcal{F}$.

Definition 5. Let $M$ be a complex manifold of dimension $\geq 2$ and let $\mathcal{F}$ be a singular holomorphic foliation on $M$. We say that a continuous hermitian form $H$ on $M$ is a $\mathcal{F}$-pseudo-metric if for any leaf $L \subset M \backslash \operatorname{sing}(\mathcal{F})$ of $\mathcal{F}$, the quadratic form $h_{L}$ defined on $L$ by the restriction $\left.H\right|_{L}$ is a conformal pseudo-riemannian metric on $L$. We say that $H$ is $\mathcal{F}$-ultrahyperbolic of curvature bounded by $a<0$ if for any leaf $L$ of $\mathcal{F}, h_{L}$ is ultrahyperbolic of curvature bounded by $a<0$. We say that $H$ is $\mathcal{F}$-ultrahyperbolic if it is $\mathcal{F}$-ultrahyperbolic of curvature bounded by $a<0$ for some $a<0$.

Before state the result let us give a definition.

Definition 6. Let $M$ and $\mathcal{F}$ be as above. Suppose that all leaves of $\mathcal{F}$ are hyperbolic. We say that $\mathcal{U}$ is normal in compact parts (briefly NCP) if $\mathcal{U}$ satisfies the following property:

(*) Any family $\mathcal{H} \subset \mathcal{U}$, such that $\{\alpha(0) ; \alpha \in \mathcal{H}\}$ is relatively compact in $M$, is normal.

In particular, if $\mathcal{U}$ is NCP, then any sequence $\left(\alpha_{n}\right)_{n \geq 1}$ in $\mathcal{U}$, such that $\left(\alpha_{n}(0)\right)_{n \geq 1}$ converges in $M$, has a convergent subsequence.

Example 1. If $M$ is complete hyperbolic in the sense of Kobayashi (cf. [K]), then all leaves of $\mathcal{F}$ are hyperbolic and $\mathcal{U}$ is NCP. In particular this is true if $M$ is a bounded ball or polydisc in $\mathbb{C}^{n}$.

The next result is a generalization of Theorems A and A.1.

Proposition 2. Let $\mathcal{F}$ be a singular holomorphic foliation on a complex manifold $M$. Let $\mu$ be a continuous $\mathcal{F}$-ultrahyperbolic hermitian metric on $M \backslash \operatorname{sing}(\mathcal{F})$. Then the following properties are true:

(a) All leaves of $\mathcal{F}$ are hyperbolic.

(b) Suppose that there exists a complete hermitian metric $g$ on $M$ such that $g \leq \mu$ on $M \backslash \operatorname{sing}(\mathcal{F})$. Then $\mathcal{U}$ is $N C P$.

In particular, any sequence $\left(\alpha_{n}\right)_{n \geq 1}$ in $\mathcal{U}$, such that the sequence $\left(\alpha_{n}(0)\right)_{n \geq 1}$ is convergent, has a convergent subsequence.

(c) If $\mu$ is complete on $M \backslash \operatorname{sing}(\mathcal{F})$ and $\operatorname{sing}(\mathcal{F})$ is discrete, then, for any convergent sequence $\left(\alpha_{n}\right)_{n \geq 1}$ in $\mathcal{U}$, we have that:

(1) If $\lim _{n \rightarrow \infty} \alpha_{n}(0)=p \in \operatorname{sing}(\mathcal{F})$, then $\left(\alpha_{n}\right)_{n \geq 1}$ converges to the constant $p$.

(2) If $\lim _{n \rightarrow \infty} \alpha_{n}(0)=p \notin \operatorname{sing}(\mathcal{F})$, then $\left(\alpha_{n}\right)_{n \geq 1}$ converges to an uniformization of $L_{p}$.

Proof. Assertion (a) is immediate from Ahlfors' Lemma. Let us suppose (b) and prove that $\mathcal{U}$ is NCP. Let $\mu$ be an $\mathcal{F}$-ultrahyperbolic metric of curvature bounded by $-a^{2}<0$ and $g$ be as in (c). Fix $\alpha \in \mathcal{U}$. It follows from Ahlfors' Lemma that

$$
\delta\left(\alpha\left(z_{2}\right), \alpha\left(z_{1}\right)\right) \leq d\left(\alpha\left(z_{2}\right), \alpha\left(z_{1}\right)\right) \leq d_{L}\left(\alpha\left(z_{2}\right), \alpha\left(z_{1}\right)\right) \leq \frac{1}{a} d_{P}\left(z_{2}, z_{1}\right),
$$

where $\delta$ and $d$ are the distances induced by $g$ and $\mu$ on $M$ and $M \backslash \operatorname{sing}(\mathcal{F})$, respectively, $d_{L}$ is the distance induced by $d$ on $L=\alpha(\mathbb{D})$ and $d_{P}$ is the Poincaré distance in $\mathbb{D}$. In particular $\mathcal{U}$ is equicontinuous with respect to $\delta$ and $d_{P}$. In order to prove that $\mathcal{U}$ is NCP, it is enough to consider the case $\mathcal{H}=\left\{\alpha_{n} ; n \geq 1\right\}$ where $\left(\alpha_{n}\right)_{n \geq 1}$ is a sequence in $\mathcal{U}$ such that $\lim _{n \rightarrow \infty} \alpha_{n}(0)=p \in M$. Let us 
prove that such sequence has a subsequence which converges in the compact parts of $\mathbb{D}$. To do that, it is enough to prove that for any fixed $0<\rho<1$ the set $V=\bigcup_{n>1} \alpha_{n}\left(\bar{D}_{\rho}\right)$ is relatively compact in $M$, where $\bar{D}_{\rho}=\{z \in \mathbb{D} ;|z| \leq \rho\}$. In fact, if this is true, it follows from the theorem of Arzela-Ascoli that the sequence of restrictions $\left(\left.\alpha_{n}\right|_{D_{\rho}}\right)_{n \geq 1}$ has a convergent subsequence, for any $0<\rho<1$. On the other hand, if we apply this fact and diagonal Cantor's method to $\left\{\left.\alpha_{n}\right|_{\bar{D}_{\rho_{m}}}\right\}_{m, n \geq 1}$, where $\rho_{m}=1-1 / m$, we get a subsequence of $\left(\alpha_{n}\right)_{n \geq 1}$, which converges in the compact parts of $\mathbb{D}$.

Let us prove that $V=\bigcup_{n \geq 1} \alpha_{n}\left(\bar{D}_{\rho}\right)$ is relatively compact in $M$. Suppose by contradiction that $V$ is not relatively compact. This implies that there exists a sequence $\left(p_{k}=\alpha_{n_{k}}\left(z_{k}\right)\right)_{k \geq 1}$ in $V$ which tends to infinite in $M$. On the other hand, it follows from (1) that

$$
\begin{aligned}
& \delta\left(\alpha_{1}(0), p_{k}\right) \leq d\left(\alpha_{1}(0), p_{k}\right) \leq d\left(\alpha_{1}(0), \alpha_{n_{k}}(0)\right)+d\left(\alpha_{n_{k}}(0), \alpha_{n_{k}}\left(z_{k}\right)\right) \\
& \leq d\left(\alpha_{1}(0), \alpha_{n_{k}}(0)\right)+\frac{1}{a} d_{P}\left(0, z_{k}\right) \leq d\left(\alpha_{1}(0), \alpha_{n_{k}}(0)\right)+\frac{1}{a} d_{P}(0, \rho) .
\end{aligned}
$$

This contradicts (b), since the set $\left\{\delta\left(\alpha_{1}(0), \alpha_{n}(0)\right) ; n \geq 1\right\}$ is bounded and $g$ is complete. Therefore $V$ is relatively compact.

Let us suppose (c). We observe that the proof of assertion (2) is similar to the proof of Theorem A of [LN], so that we will prove only assertion (1).

Let $\left(\alpha_{n}\right)_{n \geq 1}$ be a sequence in $\mathcal{U}$ such that $\lim _{n \rightarrow \infty} \alpha_{n}(0)=p$, where $p \in \operatorname{sing}(\mathcal{F})$. We will prove that in this case $\left(\alpha_{n}\right)_{n \geq 1}$ converges to $\alpha \equiv p$ in the compact parts of $\mathbb{D}$. Fix $p_{o} \in M \backslash \operatorname{sing}(\mathcal{F})$ and let

$$
\bar{B}_{r}=\left\{q \in M \backslash \operatorname{sing}(\mathcal{F}) ; d\left(q, p_{o}\right) \leq r\right\} .
$$

Since $\mu$ is complete $\bar{B}_{r}$ is compact for all $r>0$ and $\bigcup_{r>0} \bar{B}_{r}=M \backslash \operatorname{sing}(\mathcal{F})$. Let $W_{r}$ be the connected component of $M \backslash \bar{B}_{r}$ which contains $p$. Clearly $W_{r_{1}} \subset W_{r_{2}}$ if $r_{1} \geq r_{2}$. Moreover, since $\bigcap_{r>0} M \backslash \bar{B}_{r}=\operatorname{sing}(\mathcal{F})$, we have that $\bigcap_{r>0} W_{r}=\{p\}$. Therefore, it is enough to prove that given $0<\rho<1$ and $r>0$, there exists $n_{o} \geq 0$ such that if $n \geq n_{o}$, then $\alpha_{n}\left(\bar{D}_{\rho}\right) \subset W_{r}$, where $\bar{D}_{\rho}=\{z \in \mathbb{D} ;|z| \leq \rho\}$.

Fix $0<\rho<1$ and $r>0$. Let $c=a^{-1} \cdot d_{P}(0, \rho)$ and $r_{1}=c+r$. Since $\lim _{n \rightarrow \infty} \alpha_{n}(0)=p$, there exists $n_{o} \geq 0$ such that $\alpha_{n}(0) \in W_{r_{1}}$, if $n \geq n_{o}$. It follows from (1) that if $|z| \in \bar{D}_{\rho}$ and $n \geq n_{o}$, then

$$
d\left(\alpha_{n}(z), p_{o}\right) \geq d\left(\alpha_{n}(0), p_{o}\right)-d\left(\alpha_{n}(z), \alpha_{n}(0)\right)>r_{1}-a^{-1} d_{P}(z, 0) \geq r_{1}-c=r .
$$

Therefore $\alpha_{n}\left(\bar{D}_{\rho}\right) \subset M \backslash \bar{B}_{r}$. From connectedness of $\alpha_{n}\left(\bar{D}_{\rho}\right)$ it follows that $\alpha_{n}\left(\bar{D}_{\rho}\right) \subset W_{r}$.

$\S 2.3$. Properties of the function $\lambda_{\mathcal{F}, g}$. In this section we consider a singular holomorphic foliation $\mathcal{F}$ on a complex manifold $M$ and a continuous hermitian metric $g$ on $M$. As before, define

$$
\lambda_{\mathcal{F}, g}(p)=\sup \left\{\left|\alpha^{\prime}(0)\right|_{g}^{2} ; \alpha \in \mathcal{H}(\mathbb{D}, \mathcal{F}) \text { and } \alpha(0)=p\right\} .
$$

As we have seen in $\S 1, \lambda_{\mathcal{F}, g}$ satisfies the following properties:

(I) $\lambda_{\mathcal{F}, g}(p)<+\infty$ if, and only if, $L_{p}$ is hyperbolic. In this case we have $\lambda_{\mathcal{F}, g}(p)=$ $\left|\alpha^{\prime}(0)\right|^{2}$, where $\alpha: \mathbb{D} \rightarrow L_{p}$ if, and only if, $\alpha$ is an uniformization of $L_{p}$ such that $\alpha(0)=p$.

(II) If $L_{p}$ is hyperbolic, then $\frac{4 g}{\lambda_{\mathcal{F}, g}}$ induces the Poincaré metric on $L_{p}$.

(III) $\lambda_{\mathcal{F}, g}$ is lower semicontinuous. 
In general $\lambda_{\mathcal{F}, g}$ is not continuous. In this section we will see some conditions for the continuity of $\lambda_{\mathcal{F}, g}$ in $M \backslash \operatorname{sing}(\mathcal{F})$. We will assume that $\operatorname{sing}(\mathcal{F})$ is discrete and that all leaves of $\mathcal{F}$ are hyperbolic. In this case $\lambda_{\mathcal{F}, g}<+\infty$ and $\frac{4 g}{\lambda_{\mathcal{F}, g}}$ induces the Poincaré metric on the leaves of $\mathcal{F}$, so that the continuity of $\lambda_{\mathcal{F}, g}$ is equivalent to the continuity of the Poincaré metric on the leaves.

Proposition 3. Let $M, \mathcal{F}, g$ and $\lambda_{\mathcal{F}, g}$ be as before. Suppose that $\mathcal{U}$ is $N C P$. Then the following assertions are equivalent:

(a) $\lambda_{\mathcal{F}, g}$ is continuous in $M \backslash \operatorname{sing}(\mathcal{F})$.

(b) For any sequence $\left(\alpha_{n}\right)_{n \geq 1}$ in $\mathcal{U}$, which converges in the compact parts of $\mathbb{D}$ to some $\alpha: \mathbb{D} \rightarrow M$ and $p=\alpha(0) \notin \operatorname{sing}(\mathcal{F})$, then $\alpha(\mathbb{D}) \subset L_{p}$, where $L_{p}$ is the leaf of $\mathcal{F}$ through $p$.

(c) For any sequence $\left(\alpha_{n}\right)_{n \geq 1}$ in $\mathcal{U}$, which converges in the compact parts of $\mathbb{D}$ to some $\alpha: \mathbb{D} \rightarrow M$ and $p=\alpha(0) \notin \operatorname{sing}(\mathcal{F})$, then $\alpha$ is an uniformization of $L_{p}$.

(d) For any sequence $\left(\alpha_{n}\right)_{n \geq 1}$ in $\mathcal{U}$, which converges in the compact parts of $\mathbb{D}$ to some $\alpha: \mathbb{D} \rightarrow M$ and $p=\alpha(0) \in \operatorname{sing}(\mathcal{F})$, then $\alpha \equiv p$.

Proof. Let us prove first that (b), (c) and (d) are equivalent. Clearly (c) $\Longrightarrow$ (b).

(b) $\Longrightarrow(\mathbf{c})$. Suppose (b) and let $\left(\alpha_{n}\right)_{n \geq 1}$ be a sequence in $\mathcal{U}$ which converges in the compact parts of $\mathbb{D}$ to some $\alpha: \mathbb{D} \rightarrow M$, where $\alpha(0)=p \notin \operatorname{sing}(\mathcal{F})$ and $\alpha(\mathbb{D}) \subset L_{p}$. Set $\lambda=\lambda_{\mathcal{F}, g}$ and $p_{n}=\alpha_{n}(0), n \geq 1$. Let $\beta: \mathbb{D} \rightarrow L_{p}$ be an uniformization of $L_{p}$ such that $\beta(0)=p$, so that $\left|\beta^{\prime}(0)\right|_{g}^{2} \geq\left|\alpha^{\prime}(0)\right|_{g}^{2}$. Since $\lambda$ is lower semicontinuous, we have

$$
\lim _{n \rightarrow \infty} g_{p_{n}}\left(\alpha_{n}^{\prime}(0)\right)=\lim _{n \rightarrow \infty} \lambda\left(p_{n}\right)=\left|\alpha^{\prime}(0)\right|_{g}^{2} \geq \lambda(p)=\left|\beta^{\prime}(0)\right|_{g}^{2},
$$

and this implies that $\left|\alpha^{\prime}(0)\right|_{g}^{2}=\lambda(p)=\left|\beta^{\prime}(0)\right|_{g}^{2}$ and that $\alpha$ is an uniformization of $L_{p}$.

(b) $\Longrightarrow(\mathbf{d})$. We need a lemma.

Lemma 1. Let $\left(\alpha_{n}\right)_{n \geq 1}$ be a sequence in $\mathcal{U}$ which converges in the compact parts of $\mathbb{D}$ to some $\alpha: \mathbb{D} \rightarrow M$. Then $\alpha(\mathbb{D}) \subset L \cup \operatorname{sing}(\mathcal{F})$, where either $L=\emptyset$ or $L$ is a leaf of $\mathcal{F}$. If $L=\emptyset$, then $\alpha(\mathbb{D})=q \in \operatorname{sing}(\mathcal{F})$. If $L$ is a leaf of $\mathcal{F}$, then $\alpha(\mathbb{D}) \subset L \cup S$, where $S$ is some subset of $\operatorname{sing}(\mathcal{F})$ and $L$ contains at least one local separatrix of any $q \in S$.

Note. A local separatrix of a singularity $q$ of $\mathcal{F}$ is an analytic curve $\gamma$ contained in a small neighborhood $W$ of $q$ such that $q \in \gamma$ and $\gamma \backslash\{q\}$ is a leaf of $\left.\mathcal{F}\right|_{W}$ (cf. C-S]).

Proof. Suppose that $\alpha$ is not constant. Since $\operatorname{sing}(\mathcal{F})$ is discrete, it follows that $\alpha(\mathbb{D})$ contains some $p \notin \operatorname{sing}(\mathcal{F})$. Let $U=\{z \in \mathbb{D} ; \alpha(z) \notin \operatorname{sing}(\mathcal{F})\}$. Denote by $L_{z}$ the leaf of $\mathcal{F}$ through $\alpha(z)$.

Assertion 1. If $z_{o} \in U$, then the set $U_{z_{o}}=\left\{z \in U ; L_{z}=L_{z_{o}}\right\}$ is open.

Proof. Let $z_{1} \in U_{z_{o}}$. Take a local chart $(x, y): W \rightarrow D_{r} \times D_{r}^{n-1}$ around $\alpha\left(z_{1}\right)=p_{1}$, such that the leaves of $\left.\mathcal{F}\right|_{W}$ are the sets $y=$ cte and $\left(x\left(p_{1}\right), y\left(p_{1}\right)\right)=(0,0) \in$ $D_{r} \times D_{r}^{n-1}$. Let $V$ be the connected component of $\alpha^{-1}(W)$ which contains $z_{1}$. It is enough to prove that $y(\alpha(V))=\{0\}$. Suppose the opposite, by contradiction. In this case, since $y\left(\alpha\left(z_{1}\right)\right)=0$, we have that $y \circ \alpha: V \rightarrow W$ is not constant. This implies that $d(y \circ \alpha)\left(z_{2}\right) \neq 0$ for some $z_{2} \in V$ near $z_{1}$. Since $\alpha_{n}$ converges to $\alpha$, it follows that $d\left(y \circ \alpha_{n}\right)\left(z_{2}\right) \neq 0$, for $n$ big enough, so that $\alpha_{n}(\mathbb{D})$ cannot be contained in a leaf of $\mathcal{F}$, a contradiction. This proves the assertion. 
Now, let $F=\alpha^{-1}(\sin g(\mathcal{F}))$, which is a closed subset of $\mathbb{D}$. Since $\alpha$ is not constant and $\operatorname{sing}(\mathcal{F})$ is discrete, it follows that $F$ is discrete. Therefore $\mathbb{D} \backslash F=U$ is connected. It follows from the assertion that $U=U_{z_{o}}$ for some $z_{o} \in \mathbb{D}$, which proves the lemma.

Now, suppose (b) and let $\left(\alpha_{n}\right)_{n \geq 1}$ be a sequence in $\mathcal{U}$ which converges in the compact parts of $\mathbb{D}$ to some $\alpha: \mathbb{D} \rightarrow M$, where $\alpha(0)=p \in \operatorname{sing}(\mathcal{F})$. Let us prove that $\alpha \equiv p$. Suppose by contradiction that $\alpha$ is not constant. It follows from Lemma 1 that $p \in \alpha(\mathbb{D}) \subset L \cup \operatorname{sing}(\mathcal{F})$, where $L$ is some leaf of $\mathcal{F}$. Moreover $\alpha(\mathbb{D})$ contains a point $q=\alpha\left(z_{o}\right) \notin \operatorname{sing}(\mathcal{F})$. Let $\beta$ be an automorphism of $\mathbb{D}$ such that $\beta(0)=z_{o}$. Since $\lim _{n \rightarrow \infty} \alpha_{n}=\alpha$ we get $\lim _{n \rightarrow \infty} \alpha_{n} \circ \beta=\alpha \circ \beta$. On the other hand, $\alpha \circ \beta(0)=q \notin \operatorname{sing}(\mathcal{F})$, so that $\alpha \circ \beta(\mathbb{D}) \subset L$, which contradicts $p \in \alpha \circ \beta(\mathbb{D})$. Therefore $(\mathrm{b}) \Longrightarrow(\mathrm{d})$.

(d) $\Longrightarrow(\mathbf{b})$. Let $\left(\alpha_{n}\right)_{n \geq 1}$ be a sequence in $\mathcal{U}$ which converges in the compact parts of $\mathbb{D}$ to some $\alpha: \mathbb{D} \rightarrow M$, where $\alpha(0)=p \notin \operatorname{sing}(\mathcal{F})$. Let us prove that $\alpha(\mathbb{D}) \subset L_{p}$. It follows from Lemma 1 that $\alpha(\mathbb{D}) \subset L_{p} \cup \operatorname{sing}(\mathcal{F})$. Suppose by contradiction that $\alpha(\mathbb{D})$ contains some point $q \in \sin g(\mathcal{F})$. Let $z_{o} \in \mathbb{D}$ be such that $\alpha\left(z_{o}\right)=q$ and $\beta$ be an automorphism of $\mathbb{D}$ such that $\beta(0)=z_{o}$. Then $\lim _{n \rightarrow \infty} \alpha_{n} \circ \beta=\alpha \circ \beta$, where $\alpha \circ \beta(0)=q \in \operatorname{sing}(\mathcal{F})$. It follows from (d) that $\alpha \circ \beta \equiv q$, which is a contradiction. Therefore (d) $\Longrightarrow(\mathbf{b})$.

(a) $\Longrightarrow(\mathbf{b})$. Let $\left(\alpha_{n}\right)_{n \geq 1}$ be a sequence in $\mathcal{U}$ which converges in the compact parts of $\mathbb{D}$ to some $\alpha: \mathbb{D} \rightarrow M$, where $\alpha(0)=p \notin \operatorname{sing}(\mathcal{F})$. It follows from Lemma 1 that $\alpha(\mathbb{D}) \subset L_{p} \cup \operatorname{sing}(\mathcal{F})$. Suppose by contradiction that $\alpha(\mathbb{D})$ contains some nonempty subset $S$ of $\operatorname{sing}(\mathcal{F})$. Let $\tilde{S}=\alpha^{-1}(S)$ and $\tilde{\alpha}=\left.\alpha\right|_{\mathbb{D} \backslash \tilde{S}}: \mathbb{D} \backslash \tilde{S} \rightarrow L_{p}$. We need a lemma.

Lemma 2. $\tilde{\alpha}$ is a covering map.

Proof. Since $\lim _{n \rightarrow \infty} \alpha_{n}=\alpha$ and $\alpha_{n} \in \mathcal{U}$, it is not difficult to see that $\tilde{\alpha}$ is an immersion, that is, $\tilde{\alpha}^{\prime}(z) \neq 0$ for all $z \in \mathbb{D} \backslash \tilde{S}$. Therefore it is sufficient to prove that $\tilde{\alpha}$ satisfies the property of path lifting (cf. [El1]), that is, for any path $\gamma:[0,1] \rightarrow L_{p}$ such that $\gamma(0)=p$, there exists a path $\tilde{\gamma}:[0,1] \rightarrow \mathbb{D} \backslash \tilde{S}$ such that $\tilde{\gamma}(0)=0 \in \mathbb{D}$ and $\tilde{\alpha} \circ \tilde{\gamma}=\gamma$. Let us prove this fact. Fix a path $\gamma$ as before. We can suppose that $\gamma$ is of class $C^{1}$. Let $\mu=\frac{4 g}{\lambda}$. It follows from (a) that $\mu$ is continuous. Moreover $\mu$ induces the Poincaré metric on the leaves of $\mathcal{F}$. Let $k=\sup \left\{\mu_{\gamma(t)}\left((\gamma)^{\prime}(t)\right) ; t \in[0,1]\right\}$. Since $\gamma[0,1]$ is compact and does not contain singular points of $\mathcal{F}$, it is possible to find a compact neighborhood $V$ of $\gamma[0,1]$ in $L_{p}$ and a tubular neighborhood $\pi: \mathcal{W} \rightarrow V$ such that if $q \in \pi^{-1}(p)$ is near $p$, then we can lift $\gamma$ to a $C^{1}$ curve $\gamma_{q}:[0,1] \rightarrow L_{q}$ such that $\pi \circ \gamma_{q}=\gamma$, where $L_{q}$ is the leaf of $\mathcal{F}$ through $q$ (cf. [C-LN]). We have $\lim _{q \rightarrow p} \gamma_{q}=\gamma$, so that

$$
\lim _{q \rightarrow p}\left(\sup \left\{\mu_{\gamma_{q}(t)}\left(\left(\gamma_{q}\right)^{\prime}(t)\right) ; t \in[0,1]\right\}\right)=k .
$$

Since $\lim _{n \rightarrow \infty} \alpha_{n}(0)=\alpha(0)$, there exists a sequence $\left(z_{n}\right)_{n \geq n_{o}}$ in $\mathbb{D}$ such that $q_{n}=$ $\alpha_{n}\left(z_{n}\right) \in \pi^{-1}(p)$ and $\lim _{n \rightarrow \infty} z_{n}=0$. Now, $\alpha_{n}$ is a covering for all $n$. Therefore we can lift the curve $\gamma_{n}=\gamma_{q_{n}}$ to a curve $\tilde{\gamma}_{n}$ in $\mathbb{D}$ such that $\tilde{\gamma}_{n}(0)=z_{n}$, for all $n \geq n_{o}$. From (3) we can suppose that

$$
\sup \left\{\mu_{\gamma_{n}(t)}\left(\left(\gamma_{n}\right)^{\prime}(t)\right) ; t \in[0,1]\right\} \leq 2 k
$$

for all $n \geq n_{o}$. Since $\mu$ induces the Poincaré metric on the leaves of $\mathcal{F}$, this implies that the set $\left\{\ell_{P}\left(\tilde{\gamma}_{n}\right) ; n \geq n_{o}\right\}$ is bounded, where $\ell_{P}(\delta)$ denotes the length of a curve 
$\delta:[0,1] \rightarrow \mathbb{D}$ in the Poincaré metric. Now, this fact and $\lim _{n \rightarrow \infty} \tilde{\gamma}_{n}(0)=0$ imply that the sequence $\left(\tilde{\gamma}_{n}\right)_{n \geq n_{o}}$ has a convergent subsequence, say

$$
\lim _{k \rightarrow \infty} \tilde{\gamma}_{n_{k}}=\tilde{\gamma}:[0,1] \rightarrow \mathbb{D},
$$

such that $\tilde{\gamma}(0)=0$. Now, the fact that $\lim _{n \rightarrow \infty} \alpha_{n}=\alpha$ in compact parts of $\mathbb{D}$ implies that

$$
\alpha \circ \tilde{\gamma}=\lim _{k \rightarrow \infty} \alpha_{n_{k}} \circ \tilde{\gamma}_{n_{k}}=\lim _{k \rightarrow \infty} \gamma_{n_{k}}=\gamma .
$$

It follows that $\tilde{\alpha} \circ \tilde{\gamma}=\gamma$.

Let $\beta: \mathbb{D} \rightarrow L_{p}$ be a uniformization of $L_{p}$ such that $\beta(0)=p$. Since $\lambda$ is continuous we get $g_{p}\left(\alpha^{\prime}(0)\right)=\lambda(p)=g_{p}\left(\beta^{\prime}(0)\right)$.

Now, let $\pi: \mathbb{D} \rightarrow \mathbb{D} \backslash \tilde{S}$ be a uniformization of $\mathbb{D} \backslash \tilde{S}$ such that $\pi(0)=0$. Since $\tilde{S} \neq \emptyset$ we have $\left|\pi^{\prime}(0)\right|<1$ (the Schwarz Lemma). On the other hand, if $\tilde{\pi}: \mathbb{D} \rightarrow \mathbb{D}$ is a lifting of $\tilde{\alpha} \circ \pi: \mathbb{D} \rightarrow L_{p}$ by the universal covering $\beta$, such that $\tilde{\pi}(0)=0$, then we have $\left|\tilde{\pi}^{\prime}(0)\right|=1$ (because $\tilde{\alpha} \circ \pi$ is a covering map) and

$$
\left.\left.\left|\beta^{\prime}(0)\right|_{g}^{2}=\mid \beta \circ \tilde{\pi}\right)\left.^{\prime}(0)\right|_{g} ^{2}=\mid \tilde{\alpha} \circ \pi\right)\left.^{\prime}(0)\right|_{g} ^{2}=\left.\left|\tilde{\alpha}^{\prime}(0)\right|\right|_{g} ^{2}\left|\pi^{\prime}(0)\right|^{2}<\left|\tilde{\alpha}^{\prime}(0)\right|_{g}^{2}=\left|\alpha^{\prime}(0)\right|_{g}^{2}
$$

which is a contraction. This implies that $\tilde{S}=\emptyset$. Therefore (a) $\Longrightarrow($ b).

(c) $\Longrightarrow$ (a). Let $p \in M \backslash \operatorname{sing}(\mathcal{F})$ and $\left(p_{n}\right)_{n \geq 1}$ be a sequence in $M \backslash \operatorname{sing}(\mathcal{F})$ converging to $p$. Since $\lambda$ is lower semicontinuous we have $\liminf _{n \rightarrow \infty} \lambda\left(p_{n}\right) \geq \lambda(p)$. It is enough to prove that any subsequence $\left(p_{n_{k}}\right)_{k \geq 1}$ has a subsequence $\left(p_{n_{k_{\ell}}}\right)_{\ell \geq 1}$ such that $\lim _{\ell \rightarrow \infty} \lambda\left(p_{n_{k_{\ell}}}\right)=\lambda(p)$.

Let $\left(q_{k}=p_{n_{k}}\right)_{k \geq 1}$ be a subsequence of $\left(p_{n}\right)_{n \geq 1}$. Let $\left(\alpha_{k}\right)_{k \geq 1}$ be a sequence in $\mathcal{U}$ such that $\alpha_{k}(0)=q_{k}$ for all $k \geq 1$. Since $\mathcal{U}$ is NCP the sequence $\left(\alpha_{k}\right)_{k \geq 1}$ is normal, and hence it has a convergent subsequence $\left(\alpha_{k_{\ell}}\right)_{\ell \geq 1}$. It follows from (c) that $\left(\alpha_{k_{\ell}}\right)_{\ell \geq 1}$ converges to a uniformization of $L_{p}$, say $\alpha: \mathbb{D} \rightarrow L_{p}$, such that $\alpha(0)=p$. On the other hand

$$
\lim _{\ell \rightarrow \infty} \lambda\left(q_{k_{\ell}}\right)=\lim _{\ell \rightarrow \infty} g_{q_{k_{\ell}}}\left(\left(\alpha_{k_{\ell}}\right)^{\prime}(0)\right)=\left|\alpha^{\prime}(0)\right|_{g}^{2}=\lambda(p) .
$$

This finishes the proof of the proposition.

Corollary 1. Let $\mathcal{F}$ be a holomorphic foliation on a complex manifold $M$ such that $\operatorname{sing}(\mathcal{F})$ is discrete. Suppose that all leaves of $\mathcal{F}$ are hyperbolic and that $\mathcal{U}$ is $N C P$. Let $g_{1}$ and $g_{2}$ be two hermitian metrics on $M$ and $\lambda_{1}=\lambda_{\mathcal{F}, g_{1}}, \lambda_{2}=\lambda_{\mathcal{F}, g_{2}}$. If $\lambda_{1}$ is continuous, then $\lambda_{2}$ is also continuous.

The proof of the above corollary is immediate from Proposition 3.

Corollary 2. Let $V$ be a bounded Stein open subset of $\mathbb{C}^{n}, n \geq 2$, and let $\mathcal{F}$ be a singular holomorphic foliation on $V$ such that $\operatorname{sing}(\mathcal{F})$ is discrete. Let $g$ be a continuous hermitian metric on $V$ and $\lambda=\lambda_{\mathcal{F}, g}$ be as before. Then:

(a) All leaves of $\mathcal{F}$ are hyperbolic and $\mathcal{U}$ is $N C P$.

(b) $\lambda$ is continuous if, and only if, for any sequence $\left(\alpha_{n}\right)_{n \geq 1}$ in $\mathcal{U}$, which converges in the compact parts of $\mathbb{D}$ to some $\alpha: \mathbb{D} \rightarrow M$, then either $\alpha$ is an uniformization of some leaf of $\mathcal{F}$ or $\alpha$ is a constant contained in $\operatorname{sing}(\mathcal{F})$.

Proof. Since $V$ is bounded, it follows from Liouville's Theorem that every holomorphic map $f: \mathbb{C} \rightarrow V$ is constant. This implies that all leaves of $\mathcal{F}$ are hyperbolic. Moreover, Montel's Theorem implies that $\mathcal{H}(\mathbb{D}, V)$ is normal, that is, any sequence $\left(\alpha_{n}\right)_{n \geq 1}$ in $\mathcal{H}(\mathbb{D}, V)$ has a convergent subsequence, say $\lim _{k \rightarrow \infty} \alpha_{n_{k}}=\alpha: \mathbb{D} \rightarrow \bar{V}$. Now, if $\left\{\alpha_{n}(0) ; n \geq 1\right\}$ is relatively compact in $V$, then $\alpha(0) \in V$. Since $V$ is 
Stein, we must have $\alpha(\mathbb{D}) \subset V$. This implies that $\mathcal{U}$ is NCP. Part (b) follows from Proposition 3.

Corollary 3. Let $\mathcal{F}$ be a holomorphic foliation on a complex manifold $M$ such that $\operatorname{sing}(\mathcal{F})$ is discrete. Suppose that there exists on $M \backslash \operatorname{sing}(\mathcal{F})$ a continuous complete $\mathcal{F}$-ultrahyperbolic metric. Let $g$ be a hermitian metric on $M$ and $\lambda=\lambda_{\mathcal{F}, g}$ be as before. Then all leaves of $\mathcal{F}$ are hyperbolic, $\mathcal{U}$ is NCP and $\lambda$ is continuous.

The proof of the above corollary is immediate from Propositions 2 and 3.

Next we will see how the function $\lambda_{\mathcal{F}, g}$ behaves in terms of the domain in which we consider the foliation. We will consider the following situation: let $\mathcal{F}, M$ and $g$ be as before. Given a connected open subset $U$ of $M$, denote by $\mathcal{F}_{U}$ the restriction of $\mathcal{F}$ to $U$ and by $\lambda_{U}$ the function $\lambda_{\mathcal{F}_{U}, g}$.

Proposition 4. Let $M, \mathcal{F}, g$ and $\lambda_{\mathcal{F}, g}$ be as before. Suppose that all leaves of $\mathcal{F}$ are hyperbolic.

(a) Let $U \subset V$ be two connected open subsets of $M$. Then $\lambda_{U}(p) \leq \lambda_{V}(p) \leq$ $\lambda_{\mathcal{F}, g}(p)$ for all $p \in U$.

(b) Let $\left(U_{n}\right)_{n \geq 1}$ be an increasing sequence of connected open subsets of $M$ and $U=\bigcup_{n \geq 1} U_{n}$. Then $\lim _{n \rightarrow \infty} \lambda_{U_{n}}(p)=\lambda_{U}(p)$ for all $p \in U$. In particular, if all $\lambda_{U_{n}}$ and $\lambda_{U}$ are continuous, then $\left(\lambda_{U_{n}}\right)_{n \geq 1}$ converges uniformly in the compact parts of $U \backslash \operatorname{sing}(\mathcal{F})$ to $\lambda_{U}$.

Proof. Let us prove (a). Fix $p \in U$. Let $L_{U}$ and $L_{V}$ be the leaves of $\mathcal{F}_{U}$ and $\mathcal{F}_{V}$ through $p$, respectively. Let $\alpha_{U}$ and $\alpha_{V}$ be uniformizations of $L_{U}$ and $L_{V}$, respectively, such that $\alpha_{U}(0)=\alpha_{V}(0)=p$. If $i: L_{U} \rightarrow L_{V}$ is the inclusion, then we can lift $i \circ \alpha_{U}: \mathbb{D} \rightarrow L_{V}$ to a map $\beta: \mathbb{D} \rightarrow \mathbb{D}$ such that $\beta(0)=0$ and $\alpha_{V} \circ \beta=i \circ \alpha_{U}$. From the Schwarz Lemma we have $\left|\beta^{\prime}(0)\right| \leq 1$, so that

$$
\begin{aligned}
\lambda_{U}(p) & =\left|\left(i \circ \alpha_{U}\right)^{\prime}(0)\right|_{g}^{2}=\left|\left(\alpha_{V} \circ \beta\right)^{\prime}(0)\right|_{g}^{2} \\
& =\left|\alpha_{V}^{\prime}(0)\right|_{g}^{2} \cdot\left|\beta^{\prime}(0)\right|^{2} \leq\left|\alpha_{V}^{\prime}(0)\right|_{g}^{2}=\lambda_{V}(p),
\end{aligned}
$$

which proves (a).

Now, let $\left(U_{n}\right)_{n>1}$ be an increasing sequence of connected open subsets of $M$ and $U=\bigcup_{n \geq 1} U_{n}$. Set $\lambda_{U}=\lambda$ and $\lambda_{U_{n}}=\lambda_{n}$. It follows from (a) that $\lambda_{n} \leq \lambda_{n+1} \leq \lambda$, for all $n \geq 1$. Fix $p \in U$ and $n_{o}$ such that $p \in U_{n}$ for all $n \geq n_{o}$ and let us prove that $\lim _{n \rightarrow \infty} \lambda_{n}(p)=\lambda(p)$. Since $\lambda_{n}(p) \leq \lambda_{n+1}(p) \leq \lambda(p)$ for all $n \geq n_{o}$, there exists $\lim _{n \rightarrow \infty} \lambda_{n}(p)=\tilde{\lambda}(p) \leq \lambda(p)$. Denote by $L$ (resp. $L_{n}$ ) the leaf of $\mathcal{F}_{U}$ (resp. $\mathcal{F}_{U_{n}}, n \geq n_{o}$ ) through $p$. Observe that $L=\bigcup_{n \geq n_{o}} L_{n}$. In fact, it is easy to see that $L \supset \bigcup_{n>n_{o}} L_{n}$. On the other hand, if $q \in L$ there exists a curve $\gamma \subset L$ joining $p$ to $q$. Since $\gamma$ is compact, it follows that $\gamma \subset U_{n}$ for $n \geq n_{1}$, so that $\gamma \subset L_{n}$ if $n \geq n_{1}$ and $q \in \bigcup_{n \geq 1} L_{n}$. With the same type of argument, we can prove that for any compact subset $K \subset L$ there exists $n_{1} \geq n_{o}$ such that $K \subset L_{n}$ for all $n \geq n_{1}$.

Let $\alpha: \mathbb{D} \rightarrow L$ (resp. $\alpha_{n}: \mathbb{D} \rightarrow L_{n}$ ) be a uniformization of $L$ (resp. $L_{n}$ ) such that $\alpha(0)=p\left(\right.$ resp. $\left.\alpha_{n}(0)=p\right)$. Fix $0<r<1$ and let $\overline{D_{r}}=\{z ;|z| \leq r\}$. Since $\alpha\left(\overline{D_{r}}\right)$ is compact, there exists $n_{r} \geq n_{o}$ such that $\alpha\left(\overline{D_{r}}\right) \subset L_{n}$ for all $n \geq n_{r}$. For each $n \geq n_{r}$, let $\beta_{n}: D_{r} \rightarrow \mathbb{D}$ be a lifting of $\left.\alpha\right|_{D_{r}}: D_{r} \rightarrow L_{n}$, such that $\alpha_{n} \circ \beta_{n}=\left.\alpha\right|_{D_{r}}$ and $\beta_{n}(0)=0$. It follows from the Schwarz Lemma that $\left|\beta_{n}^{\prime}(0)\right| \leq \frac{1}{r}$. On the other hand,

$$
\frac{1}{r^{2}} \lambda_{n}(p) \geq\left|\alpha_{n}^{\prime}(0)\right|^{2}\left|\beta_{n}^{\prime}(0)\right|^{2}=\left|\left(\alpha_{n} \circ \beta_{n}\right)^{\prime}(0)\right|_{g}^{2}=\left|\alpha^{\prime}(0)\right|_{g}^{2}=\lambda(p),
$$


which implies that $\tilde{\lambda}(p) \geq r^{2} \lambda(p)$. Since $0<r<1$ is arbitrary, it follows that $\tilde{\lambda}(p) \geq \lambda(p)$, and so $\tilde{\lambda}(p)=\lambda(p)$. The last assertion in (b) follows from Dini's Theorem.

Corollary. Let $\mathcal{F}$ be a germ of foliation at $0 \in \mathbb{C}^{n}$. If $\mathcal{F}$ satisfies property P.2, then it satisfies property $\mathbf{P . 1 .}$

Proof. Let $g=|d z|^{2}$ be the euclidean metric on $\mathbb{C}^{n}$. Since $\mathcal{F}$ satisfies property P.2, there exists a neighborhood $U$ of 0 such that $\mathcal{F}$ is defined in $U$ and $\mu_{U}=\frac{4 g}{\lambda_{U}}$ is complete at 0 , where $\lambda_{U}=\lambda_{\left.\mathcal{F}\right|_{U}, g}$. Let $B=B_{r}=\left\{z \in \mathbb{C}^{n} ;|z|<r\right\} \subset U$ and $\mu_{B}=\frac{4 g}{\lambda_{B}}$. It follows from Proposition 4 that $\mu_{B} \geq \mu_{U}$, and so $\mu_{B}$ is complete at 0 . Let us prove that $\lambda_{B}$ is continuous. Since $B$ is Stein, it follows from Corollary 2 of Proposition 3 that it is enough to prove that if $\left(\alpha_{n}\right)_{n \geq 1}$ is a sequence in $\mathcal{U}$, which converges in the compact parts of $\mathbb{D}$ to some $\alpha: \mathbb{D} \rightarrow M$, where $\alpha(0)=0 \in \mathbb{C}^{n}$, then $\alpha \equiv 0$. On the other hand, the proof of this fact is similar to the proof of (c)(1) in Proposition 2. We leave the details for the reader.

\section{$\S 3$. Proof of THE THEOREMS}

§3.1. Estimates in the local case. Proof of Theorems 2 and 3. In this section we will consider a germ of foliation $\mathcal{F}$ at $\left(\mathbb{C}^{n}, 0\right)$, given by a germ of holomorphic vector field $X$ with an isolated singularity at $0 \in \mathbb{C}^{n}$. Let $e=|d z|^{2}=\sum_{j=1}^{n}\left|d z_{j}\right|^{2}$ be the euclidean metric on $\mathbb{C}^{n}$. Given a neighborhood $U$ of 0 where we can define a representative $\mathcal{F}_{U}$ of $\mathcal{F}$, we will denote by $\lambda_{U}$ the function $\lambda_{\mathcal{F}_{U}, e}$ and by $\mu_{U}$ the metric $\frac{4|d z|^{2}}{\lambda_{U}}$. In most cases we will suppose that $U$ is either a ball $B_{r}=\{z=$ $\left.\left(z_{1}, \ldots, z_{n}\right) \in \mathbb{C}^{n} ;|z|^{2}=\sum_{j=1}^{n}\left|z_{j}\right|^{2}<r^{2}\right\}$, or a polidisk $P_{r}=\left\{z \in \mathbb{C}^{n} ;\left|z_{j}\right|<r\right\}$. In what follows, we are going to estimate the growth of $\lambda_{U}$ and $\mu_{U}$ when $z \rightarrow 0$. The next proposition implies Theorem 2.

Proposition 5. Let $\mathcal{F}$ and $X$ be germs as before. Suppose that $X=X_{k}+$ h.o.t., where the components of $X_{k}$ are homogeneous polynomials of degree $k, 0$ is an isolated singularity of $X_{k}$, and h.o.t. means "higher order terms". If $U=B_{\rho}$, where $X$ is defined in $B_{\rho}, \lambda_{\rho}=\lambda_{U}$ and $\mu_{\rho}=\mu_{U}$, then there exist $r>\rho$ and $K>0$ such that

$$
\mu_{\rho} \geq K \frac{|d z|^{2}}{|z|^{2}\left(\ln \left(\frac{|z|}{r}\right)\right)^{2}}, \forall z \in B_{\rho} .
$$

In particular $\mathcal{F}$ satisfies properties $\mathbf{P . 1}$ and $\mathbf{P . 2}$ at 0 .

Proof. Consider the hermitian metric $h$, defined on $B_{r} \backslash\{0\}$ by

$$
h_{z}=\frac{|z|^{(2 k-2)}|d z|^{2}}{|X(z)|^{2} \ln ^{2}(|z| / r)}
$$

where $r>\rho$ and $|z|^{2}=\sum_{j=1}^{n}\left|z_{j}\right|^{2}$. Let us compute $k_{h}$, the gaussian curvature of the leaves of $\left.\mathcal{F}\right|_{B_{\rho}}$. Fix $z \in B_{\rho} \backslash\{0\}$, and let $Z(t)$ be the solution of $\frac{d Z}{d t}=X(Z)$ such that $Z(0)=z$, which can be defined in a disk $D_{\epsilon}=\{t \in \mathbb{C} ;|t|<\epsilon\}$. If we consider the leaf of $\mathcal{F}$ through $z$ locally parametrized by $t \rightarrow Z(t)$, then $Z^{*}(h)=\phi(t)|d t|^{2}$, where

$$
\phi(t)=\frac{|Z(t)|^{(2 k-2)}\left|Z^{\prime}(t)\right|^{2}}{|X(Z(t))|^{2} \ln ^{2}(|Z(t)| / r)}=\frac{|Z(t)|^{(2 k-2)}}{\ln (|Z(t)| / r)} .
$$


It follows that

$k_{h}(Z(t))=-\frac{2}{\phi(t)} \frac{\partial^{2}}{\partial t \partial \bar{t}} \ln (\phi(t))=-\frac{2}{\phi(t)} \frac{\partial^{2}}{\partial t \partial \bar{t}}\left[(k-1) \ln \left(|Z(t)|^{2}\right)-\ln \left(\ln ^{2}(|Z(t)| / r)\right)\right]$.

By a direct computation, we get

$$
\begin{gathered}
-k_{h}(z)=-k_{h}(Z(0)) \\
=2(k-1) \frac{\ln ^{2}\left(\frac{|z|}{r}\right)}{|z|^{2 k}}\left|X(z) \wedge \frac{z}{|z|^{2}}\right|^{2}+\frac{1}{|z|^{2 k}}\left[\left|\left\langle X(z), \frac{z}{|z|}\right\rangle\right|^{2}+\left|\ln \left(\frac{|z|^{2}}{r^{2}}\right)\right|\left|X(z) \wedge \frac{z}{|z|}\right|^{2}\right],
\end{gathered}
$$

where above we use the notation $\langle z, w\rangle=\sum_{j=1}^{n} z_{j} \cdot \overline{w_{j}}$ and $|z \wedge w|^{2}=|z|^{2} \cdot|w|^{2}-$ $|\langle z, w\rangle|^{2}$. In particular, since $r>\rho$, we have $k_{h}(z)<0$ for all $z \in \overline{B_{\rho}} \backslash\{0\}$. Let us prove that if $\rho$ is small enough, then there exists $a>0$ such that $k_{h}(z)<-a$ for all $z \in B_{\rho} \backslash\{0\}$.

Since $X=X_{k}+$ h.o.t., where $X_{k}$ is homogeneous of degree $k$ and 0 is an isolated singularity of $X_{k}$, there exists $C>1$ and $\rho>0$ such that if $|z|<\rho$, then $C^{-1}|z|^{k} \leq$ $|X(z)| \leq C \cdot|z|^{k}$. On the other hand, if $|z|<\frac{r}{\sqrt{e}}$, we have

$$
\begin{gathered}
\left|\ln \left(\frac{|z|^{2}}{r^{2}}\right)\right|>1 \Longrightarrow\left|\left\langle X(z), \frac{z}{|z|}\right\rangle\right|^{2}+\left|\ln \left(\frac{|z|^{2}}{r^{2}}\right)\right|\left|X(z) \wedge \frac{z}{|z|}\right|^{2} \\
\geq\left|\left\langle X(z), \frac{z}{|z|}\right\rangle\right|^{2}+\left|X(z) \wedge \frac{z}{|z|^{2}}\right|^{2}=|X(z)|^{2}
\end{gathered}
$$

so that

$$
-k_{h}(z) \geq \frac{|X(z)|^{2}}{|z|^{2 k}} \geq C^{-2}
$$

if $|z|<\min \left\{\rho, \frac{r}{\sqrt{e}}\right\}$. This implies that $\limsup _{z \rightarrow 0} k_{h}(z) \leq-C^{-2}<0$. Since $k_{h}(z)<0$ for all $z \in \overline{B_{\rho}} \backslash\{0\}$, it follows that $k_{h}<-a$ on $B_{\rho} \backslash\{0\}$, for some $a>0$.

Let $\lambda_{\rho}$ be such that $\mu_{\rho}=\frac{4|d z|^{2}}{\lambda_{\rho}}$ induces the Poincaré metric on the leaves of $\mathcal{F}_{\rho}=\left.\mathcal{F}\right|_{B_{\rho}}$. If $L_{z}$ is the leaf of $\mathcal{F}_{\rho}$ through $z \in B_{\rho} \backslash\{0\}$, it follows from Ahlfor's Lemma that

$$
\begin{aligned}
\left.h\right|_{L_{z}} \leq\left.\frac{1}{a} \mu_{\rho}\right|_{L_{z}} & \Longrightarrow \frac{|z|^{(2 k-2)}}{|X(z)|^{2} \ln ^{2}\left(\frac{|z|}{r}\right)} \leq \frac{4}{a \lambda_{\rho}(z)} \\
& \Longrightarrow \lambda_{\rho}(z) \leq \frac{4|X(z)|^{2} \ln ^{2}\left(\frac{|z|}{r}\right)}{a|z|^{(2 k-2)}} \leq \frac{4}{a} C^{2}|z|^{2} \ln ^{2}\left(\frac{|z|}{r}\right) \\
& \Longrightarrow \mu_{\rho} \geq K \frac{|d z|^{2}}{|z|^{2}\left(\ln \left(\frac{|z|}{r}\right)\right)^{2}}, \forall z \in B_{\rho}
\end{aligned}
$$

where $K=\frac{a}{C^{2}}$.

It remains to prove that $\mathcal{F}$ satisfies property $\mathbf{P . 2}$ at 0 (cf. the Corollary of Proposition 4). This follows from the fact that the metric $\frac{|d z|^{2}}{|z|^{2}\left(\ln \left(\frac{|z|}{r}\right)\right)^{2}}$ is complete at 0 (cf. [LN]).

Now we prove a result that will be used to obtain estimates for $\frac{4|d z|^{2}}{\lambda}$ in the other direction. This result will be also used in the next section to study an example that does not satisfy Property P.2.

Let us consider the partial order relation $\preceq$ on $\mathbb{R}^{n}$, defined by

$$
x=\left(x_{1}, \ldots, x_{n}\right) \preceq y=\left(y_{1}, \ldots, y_{n}\right) \Leftrightarrow x_{j} \leq y_{j}, \forall j=1, \ldots, n .
$$


We will use the notation $\mathbb{R}_{+}^{n}=\left\{\left(x_{1}, \ldots, x_{n}\right) ; x_{j} \geq 0, \forall j=1, \ldots, n\right\}$.

Proposition 6. Let $F:[0, r] \times \mathbb{R}_{+}^{n} \rightarrow \mathbb{R}_{+}^{n}$ be a continuous function with the following properties:

(a) If $t \in[0, r]$ and $x \preceq y, x, y \in \mathbb{R}_{+}^{n}$, then $F(t, x) \preceq F(t, y)$.

(b) The differential equation $\frac{d X}{d t}=F(t, X)$ with initial condition $X(0)=$ $\left(x_{1}, \ldots, x_{n}\right) \in \mathbb{R}_{+}^{n}$ has a unique solution.

Let $x:[0, r] \rightarrow \mathbb{R}_{+}^{n}$ be a continuous function which satisfies the following inequality:

$$
x(t) \preceq x_{o}+\int_{0}^{t} F(s, x(s)) d s, \forall t \leq r,
$$

where $x_{o} \in \mathbb{R}_{+}^{n}$. Let $y:[0, \alpha) \rightarrow \mathbb{R}_{+}^{n}$ be the solution of

$$
\frac{d y}{d t}=F(t, y), y(0)=x_{o},
$$

where $[0, \alpha)$ is maximal interval of definition of $y$. Then

$$
x(t) \preceq y(t), \forall t \in\left[0, \alpha^{\prime}\right),
$$

where $\alpha^{\prime}=\min \{r, \alpha\}$. If $\alpha^{\prime}=r$, then (I) is true for all $t \in[0, r]$.

Proof. Let $\epsilon=\left(\epsilon_{1}, \ldots, \epsilon_{n}\right)$ be such that $\epsilon_{j}>0$ for all $j=1, \ldots, n$ and let $y_{\epsilon}:\left[0, \alpha_{\epsilon}\right) \rightarrow$ $\mathbb{R}_{+}^{n}$ be the solution of $\frac{d Y}{d t}=F(t, Y), Y(0)=x_{o}+\epsilon$, where $\left[0, \alpha_{\epsilon}\right)$ is the maximal interval of definition of $y_{\epsilon}$. Let $\alpha_{\epsilon}^{\prime}=\min \left\{r, \alpha_{\epsilon}\right\}$. It is clear that $x_{o}=x(0) \prec$ $y_{\epsilon}(0)=x_{o}+\epsilon$. Let

$$
A_{\epsilon}=\left\{t \in\left[0, \alpha_{\epsilon}^{\prime}\right) ; x(s) \preceq y_{\epsilon}(s) \forall s \in[0, t)\right\} .
$$

Since $x(0) \prec y_{\epsilon}(0)$, there exists $\delta>0$ such that $[0, \delta) \subset A_{\epsilon}$. Therefore $A_{\epsilon}$ is a nonempty interval of the type $[0, a)$ or $[0, a]$, where $a \leq \alpha_{\epsilon}^{\prime}, a=\sup A_{\epsilon}$. We assert that $a=\alpha_{\epsilon}^{\prime}$.

In fact, suppose by contradiction that $a<\alpha_{\epsilon}^{\prime}$. We have

$$
y_{\epsilon}(t)-x(t) \succeq \epsilon+\int_{0}^{t}\left[F\left(s, y_{\epsilon}(s)\right)-F(s, x(s))\right] d s, \forall t \in\left[0, \alpha_{\epsilon}^{\prime}\right) .
$$

On the other hand,

$$
s<\alpha_{\epsilon}^{\prime} \Longrightarrow x(s) \preceq y_{\epsilon}(s) \Longrightarrow 0 \preceq F\left(s, y_{\epsilon}(s)\right)-F(s, x(s)) .
$$

Hence, if $t<\alpha_{\epsilon}^{\prime}$, we have $0 \preceq \epsilon \preceq y_{\epsilon}(t)-x(t)$, and so $a=\alpha_{\epsilon}^{\prime}$. Now, let $t_{o}=\alpha^{\prime}-\delta$. Since $y(t)$ is defined in $\left[0, t_{o}\right]$, there exists $\delta^{\prime}>0$ such that if $|\epsilon|<\delta^{\prime}$, then $y_{\epsilon}$ is also defined in $\left[0, t_{o}\right]$. Therefore $x(t) \preceq y_{\epsilon}(t)$ for $|\epsilon|<\delta^{\prime}$ and $t \in\left[0, t_{o}\right]$. Since $\lim _{\epsilon \rightarrow 0} y_{\epsilon}(t)=y(t)$, we obtain $x(t) \preceq y(t)$, for all $t \in\left[0, t_{o}\right]$. Since $t_{o}=\alpha^{\prime}-\delta$ and $\delta$ are arbitrary, we have that $x(t) \preceq y(t), t \in\left[0, \alpha^{\prime}\right)$. It is clear that if $\alpha^{\prime}=0$, then $x(t) \preceq y(t)$ in $[0, r]$.

Remark 7. It is not difficult to see that Gronwall's Lemma (cf. $[\mathrm{M}-\mathrm{P}]$ ) is a consequence of Proposition 6.

In the next result we will use Proposition 6 to obtain estimates for $\mu_{\rho}=\frac{4|d z|^{2}}{\lambda_{\rho}}$ near 0 , in the other direction. This will finish the proof of Theorem 3. 
Proposition 7. Let $\mathcal{F}$ and $X=X_{k}+$ h.o.t. be germs as in Proposition 5. Let $U=B_{\rho}$, where $X$ is defined in $B_{\rho}, \lambda_{\rho}=\lambda_{U}, \mu_{\rho}=\mu_{U}$ and $r>\rho$. If $k=1$, then there exist $K>0$ and $0<s<\rho$ such that

$$
\mu_{\rho} \leq K \frac{|d z|^{2}}{|z|^{2}\left(\ln \left(\frac{|z|}{r}\right)\right)^{2}}, \forall z \in B_{s} .
$$

If $k>1$, then there exist $K>0$ and $0<s<\rho$ such that

$$
\mu_{\rho} \leq K \frac{|d z|^{2}}{|z|^{2}}, \forall z \in B_{s} .
$$

Proof. We need a lemma.

Lemma 3. Let $\mathcal{F}$ be a holomorphic foliation on a complex manifold $M$ whose leaves are hyperbolic, and let $g$ be a hermitian metric on $M$ and $\lambda=\lambda_{\mathcal{F}, g}$. Fix $p \in M \backslash \operatorname{sing}(\mathcal{F})$ and a holomorphic vector field $X$, which represents $\mathcal{F}$ in an open set $p \in U \subset M$. Let $z(t)$ be the complex solution of $\frac{d z}{d t}=X(z)$ with initial condition $z(0)=p$. Suppose that $z(t)$ can be extended to a disk $D_{b}=\{z \in \mathbb{C} ;|z|<b\}$. Then $\lambda(p) \geq b^{2} \cdot|X(p)|_{g}^{2}$.

Proof. Let $\alpha$ be a uniformization of $L_{p}$ such that $\alpha(0)=p$. Consider the lifting of $\beta: D_{b} \rightarrow \mathbb{D}$ by $\alpha$ such that $\beta(0)=0$ and $\alpha \circ \beta=z$. It follows from the Schwarz Lemma that $\left|\beta^{\prime}(0)\right| \leq \frac{1}{b}$. On the other hand,

$$
|X(p)|_{g}^{2}=\left|z^{\prime}(0)\right|_{g}^{2}=\left|\beta^{\prime}(0)\right|^{2} \cdot\left|\alpha^{\prime}(0)\right|_{g}^{2} \leq \frac{1}{b^{2}} \lambda(p),
$$

and so $\lambda(p) \geq b^{2} \cdot|X(p)|_{g}^{2}$.

Let us continue the proof of the proposition. Since $X=X_{k}+$ h.o.t., there exists $0<s<\rho$ such that $C^{-1} \cdot|z|^{k} \leq|X(z)| \leq C$. $|z|^{k}$, for all $z \in B_{s}$, where $C>1$. Fix $z \in B_{s} \backslash\{0\}$ and let $Z(T)$ be the solution of the complex equation $\frac{d Z}{d T}=X(Z)$ with initial condition $Z(0)=z$.

Assertion. If $k=1$, then the solution $Z(T)$ can be extended to the disk of radius

$$
R_{1}(z)=\frac{1}{C} \ln \left(\frac{s}{|z|}\right) \text {. }
$$

If $k>1$, then $Z(T)$ can be extended to the disk of radius

$$
R_{k}(z)=\frac{s^{k-1}-|z|^{k-1}}{(k-1) C|z|^{k-1} s^{k-1}}=\frac{1}{C(k-1)}\left[\frac{1}{|z|^{k-1}}-\frac{1}{s^{k-1}}\right] .
$$

Proof. Fix $w$ with $|w|=1$ and let $W(t)=Z(t . w), t \in \mathbb{R}$. Then $W$ is a solution of the real differential equation $\frac{d W}{d t}=w \cdot X(W)$ with initial condition $W(0)=z$. Let $[0, a)$ be the maximal positive interval of definition of this solution. It is enough to prove that $a \geq R_{k}(z)$ for all $w$ with $|w|=1$.

We have

$$
W(t)=z+\int_{0}^{t} w \cdot X(W(\tau)) d \tau \Longrightarrow|W(t)| \leq|z|+\int_{0}^{t}|X(W(\tau))| d \tau .
$$

Let $[0, b)$ be the maximal positive interval $(b \leq a)$, such that $|W(t)|<s$ for all $t \in[0, b)$. If $t \in[0, b)$, then

$$
|W(t)| \leq|z|+\int_{0}^{t}|X(W(\tau))| d \tau \leq|z|+\int_{0}^{t} C|W(\tau)|^{k} d \tau .
$$


Let us consider the case $k=1$. In this case, $y(t)=|z| e^{C t}$ is the solution of $\frac{d y}{d t}=C y$ with $y(0)=|z|$. It follows from Proposition 6 that $|W(t)| \leq|z| e^{C t}$ for all $t \in[0, b)$, so that if $|z| e^{C t}<s$, then, a fortiori, $|W(t)|<s$. This implies that if $t \leq \frac{1}{C} \ln \left(\frac{s}{|z|}\right)$, then $|W(t)| \in B_{s}$. It follows that $b \geq \frac{1}{C} \ln \left(\frac{s}{|z|}\right)=R_{1}(z)$.

Let us consider the case $k>1$. In this case, the solution of $\frac{d y}{d t}=C \cdot y^{k}$ with $y(0)=|z|$ is

$$
y(t)=\frac{|z|}{\left[1-(k-1) C|z|^{(k-1)} t\right]^{\frac{1}{k-1}}},
$$

so that $|W(t)| \leq y(t)$. On the other hand, the solution of the inequality $y(t)<s$ is

$$
t<\frac{s^{k-1}-|z|^{k-1}}{(k-1) C|z|^{k-1} s^{k-1}}=R_{k}(z) .
$$

This implies that $b \geq R_{k}(z)$, and proves the assertion.

Let us finish the proof of Proposition 7 . Since $\lambda=\lambda_{\mathcal{F}_{U}, e}$, we get from Lemma 3 that

$$
\lambda(z) \geq R_{k}^{2}(z) \cdot|X(z)|^{2} \geq C^{-2} \cdot R_{k}^{2}(z) \cdot|z|^{2 k},
$$

for any $z \in B_{s} \backslash\{0\}$. Let us consider the case $k=1$. In this case $R_{1}(z)=\frac{1}{C} \ln \left(\frac{s}{|z|}\right)$, so that $\lambda(z) \geq C^{-4} \cdot \ln ^{2}\left(\frac{s}{|z|}\right) \cdot|z|^{2}$. Since $\lambda>0$ in $\overline{B_{s}} \backslash\{0\}, \lambda$ is continuous and $\lim _{z \rightarrow 0}\left(\ln ^{2}\left(\frac{s}{|z|}\right)\right) /\left(\ln ^{2}\left(\frac{r}{|z|}\right)\right)=1$, it is not difficult to see that there exists $K>0$ such that $\lambda(z) \geq 4 K^{-1} \cdot \ln ^{2}\left(\frac{r}{|z|}\right) \cdot|z|^{2}$, for all $z \in B_{s} \backslash\{0\}$, which implies that

$$
\mu=\frac{4|d z|^{2}}{\lambda(z)} \leq K \frac{|d z|^{2}}{|z|^{2}\left(\ln \left(\frac{|z|}{r}\right)\right)^{2}}, \forall z \in B_{s} \backslash\{0\},
$$

which proves (a). In the case $k>1$ we have,

$$
\lambda(z) \geq C^{-2} \cdot R_{k}^{2}(z) \cdot|z|^{2 k}=C^{-4}(k-1)^{-2}|z|^{2}\left[1-\frac{|z|^{k-1}}{s^{k-1}}\right]^{2},
$$

for all $z \in B_{s} \backslash\{0\}$. Since $\lambda>0$ in $\overline{B_{s}} \backslash\{0\}, \lambda$ is continuous and $\lim _{z \rightarrow 0}\left[1-\frac{|z|^{k-1}}{s^{k-1}}\right]^{2}=$ 1 , it is not difficult to see that there exists $K>0$ such that $\lambda(z) \geq 4 K^{-1} \cdot|z|^{2}$, for all $z \in B_{s} \backslash\{0\}$, which implies that

$$
\mu=\frac{4|d z|^{2}}{\lambda(z)} \leq K \frac{|d z|^{2}}{|z|^{2}}, \forall z \in B_{s} \backslash\{0\}
$$

which proves (b).

Example 2. Let $X_{k}$ be a homogeneous vector field of degree $k \geq 2$ in $\mathbb{C}^{n}, n \geq 2$, with an isolated singularity at $0 \in \mathbb{C}^{n}$. Let $\mathcal{F}$ be the foliation defined by $X_{k}$ on the ball $B_{1}=\{z ;|z|<1\}$. In this example we will see that if we assume that $X_{k}$ is generic, then the estimates in (b) of Theorem 3 are the best possible. We will see that in some directions the order of growth of $\mu$ is exactly $\frac{|d z|^{2}}{|z|^{2}(\ln |z|)^{2}}$, whereas in other directions its order is $\frac{|d z|^{2}}{|z|^{2}}$.

Consider first a direction $z_{o} \in \partial B_{1}$ such that $X_{k}\left(z_{o}\right)=a . z_{o}, a \neq 0$. In this case $\mathcal{F}$ has a leaf $L$ of the form $L=\left\{w . z_{o} ; w \in \mathbb{C}, 0<|w|<1\right\}$, that is, $L \simeq \mathbb{D}^{*}=$ $\{w \in \mathbb{C} ; 0<|w|<1\}$. Since the Poincaré metric in $\mathbb{D}^{*}$ is $\frac{|d w|^{2}}{|w|^{2}(\ln (|w|))^{2}}(\mathrm{cf} .[\mathrm{K}])$ and 
$\mu$ induces the Poincaré metric on $L$, it follows that $\left.\mu\right|_{L}=\left.\frac{|d z|^{2}}{|z|^{2}(\ln (|z|))^{2}}\right|_{L}$. Therefore, in this direction the order of growth of $\mu$ is exactly $\frac{|d z|^{2}}{|z|^{2}(\ln (|z|))^{2}}$.

Consider now a direction $z_{1} \in \partial B_{1}$ such that $X_{k}\left(z_{1}\right) \neq 0$ and is not parallel to $z_{1}$. Denote by $\mathcal{F}_{k}$ the foliation defined by $X_{k}$ on $\mathbb{C}^{n}$ and by $\tilde{L}_{z}$ the leaf of $\mathcal{F}_{k}$ through $z \in \mathbb{C}^{n} \backslash\{0\}$. Let $H_{t}(z)=t . z, t \in \mathbb{C}, z \in \mathbb{C}^{n}$. Observe first that, since $X_{k}$ is homogeneous, then $H_{t}\left(\tilde{L}_{z}\right)=\tilde{L}_{t z}$, for all $t \in \mathbb{C}^{*}$ and $z \in \mathbb{C}^{n} \backslash\{0\}$. We will see at the end that, under generic conditions on $X_{k}$, the leaf $\tilde{L}_{z_{1}}\left(z_{1}\right.$ as above) is uniformized by $\mathbb{D}$. So, suppose that $\tilde{L}_{z_{1}}$ is hyperbolic. Given $w \in \mathbb{C}$ and $0<|w|<1$, denote by $L_{w}$ the leaf of $\mathcal{F}$ through $z=w . z_{1} \in B_{1}$. Let $\alpha_{w}: \mathbb{D} \rightarrow L_{w}$ and $\alpha: \mathbb{D} \rightarrow \tilde{L}_{z_{1}}$ be uniformizations of $L_{w}$ and $\tilde{L}_{z_{1}}$ such that $\alpha_{w}(0)=w \cdot z_{1}$ and $\alpha(0)=z_{1}$, respectively. As we have seen $H_{w^{-1}}\left(L_{w}\right) \subset \tilde{L}_{z_{1}}$, so that we can consider the lifting $\beta: \mathbb{D} \rightarrow \mathbb{D}$ of $H_{w^{-1}} \circ \alpha_{w}: \mathbb{D} \rightarrow \tilde{L}_{z_{1}}$, such that $\alpha \circ \beta=H_{w^{-1}} \circ \alpha_{w}$ and $\beta(0)=0$. This implies that $\beta^{\prime}(0) \cdot \alpha^{\prime}(0)=w^{-1} \cdot \alpha_{w}^{\prime}(0)$ and so

$$
\lambda\left(w . z_{1}\right)=\left|\alpha_{w}^{\prime}(0)\right|^{2}=|w|^{2} \cdot\left|\beta^{\prime}(0)\right|^{2} \cdot\left|\alpha^{\prime}(0)\right|^{2} \leq C \cdot|w|^{2},
$$

where $C=\left|\alpha^{\prime}(0)\right|^{2}$ and $\left|\beta^{\prime}(0)\right| \leq 1$ by the Schwarz Lemma. This implies that

$$
\mu_{w . z_{1}} \geq 4 C^{-1} \frac{|d z|^{2}}{\left|w . z_{1}\right|^{2}}
$$

which proves that the order of growth is $\frac{|d z|^{2}}{|z|^{2}}$ in the direction of $z_{1}$.

It remains to prove that generically the leaf $\tilde{L}_{z_{1}}$ is hyperbolic. Consider first the case $n=2$. Let $\pi: \mathbb{C}^{2} \backslash\{0\} \rightarrow \mathbb{C} P(1)$ be the canonical projection. Consider the set

$$
\mathcal{P}=\left\{\pi(z) ; z \in \mathbb{C}^{2} \backslash\{0\} \text { and } X_{k}(z) \text { is parallel to } z\right\} .
$$

Since $k \geq 2$, under generic conditions on $X_{k}$, we have that $\mathcal{P}$ is finite and has $N \geq 3$ elements. In this case $\mathcal{F}_{k}$ is transversal to the fibers of $\pi$ in the open set $V=\mathbb{C}^{2} \backslash \pi^{-1}(\mathcal{P})$. This implies that if $\tilde{L}_{z}$ is a leaf of $\mathcal{F}_{k}$, where $z \in V$, then $\left.\pi\right|_{\tilde{L}_{z}}: \tilde{L}_{z} \rightarrow \mathbb{C} P(1) \backslash \mathcal{P}$ is a covering map. Since the universal covering of $\mathbb{C} P(1) \backslash \mathcal{P}$ is $\mathbb{D}$, it follows that $\tilde{L}_{z}$ is hyperbolic.

Suppose now that $n \geq 3$. In this case, let $M=\left(\mathbb{C}^{n} \backslash\{0\}\right) \cup \mathbb{C} P(n-1)$ be the complex manifold obtained by blowing-up once at $0 \in \mathbb{C}^{n}, P: M \rightarrow \mathbb{C}^{n}$ be the blowing-up map and $\pi: \mathbb{C}^{n} \backslash\{0\} \rightarrow \mathbb{C} P(n-1)$ be the canonical projection. Then $\pi$ lifts to a projection $\pi^{*}: M \rightarrow \mathbb{C} P(n-1)=D$ such that $\pi \circ P=\pi^{*}$, in such a way that $\left(M, \pi^{*}, D\right)$ is a line bundle. Let $\mathcal{F}^{*}=P^{*}\left(\mathcal{F}_{k}\right)$ be the strict transform of $\mathcal{F}_{k}$ by $P$. Under generic conditions on $X_{k}$, the divisor $D=\mathbb{C} P(n-1)$ is invariant by $\mathcal{F}^{*}$ and the foliation $\left.\mathcal{F}^{*}\right|_{D}$ is a foliation of degree $k$ on $D=\mathbb{C} P(n-1)$, whose singularities are nondegenerated, that is, with Milnor's number 1 . Since $k \geq 2$, it follows from Theorem B of [LN] that all leaves of $\left.\mathcal{F}^{*}\right|_{D}$ are hyperbolic. On the other hand, if $\left.V=\pi^{-1}(\mathbb{C} P(n-1)) \backslash \operatorname{sing}\left(\left.\mathcal{F}^{*}\right|_{D}\right)\right)$ and $\tilde{L}$ is a leaf of $\left.\mathcal{F}_{k}\right|_{V}$, then $\pi(\tilde{L})=L$, a leaf of $\left.\mathcal{F}^{*}\right|_{D}$, and $\left.\pi\right|_{\tilde{L}}: \tilde{L} \rightarrow L$ is a covering map. Since $L$ is hyperbolic, it follows that $\tilde{L}$ is also hyperbolic. We leave the details for the reader.

§3.2. An example which does not satisfy Property P.2. In this section we consider the foliation $\mathcal{F}$ defined by the level surfaces of $G(x, y)=y^{p}-x^{q}$ on the polydisk $P=\left\{(x, y) \in \mathbb{C}^{2} ;|x|<1,|y|<1\right\}$, where $1<p<q$. We will prove that $\frac{4|d z|^{2}}{\lambda_{P}}$ is not complete at 0 and that the germ of $\mathcal{F}$ at 0 does not satisfy property P.2. 
Assertion. There exists a constant $a>0$ such that $\lambda_{P}(0, y) \geq a \cdot|y|^{2 p / q}$ if $|y| \leq 1 / 2$.

Proof. The leaves of $\mathcal{F}$ are the integrals of the complex equation

$$
\frac{d x}{d T}=\frac{\partial G}{\partial y}=p y^{p-1}, \frac{d y}{d T}=-\frac{\partial G}{\partial x}=q x^{q-1} .
$$

Let $\tilde{X}(x, y)=p y^{p-1} \partial x+q x^{q-1} \partial y$. Fix a point $\left(0, y_{o}\right), 0<\left|y_{o}\right|<1$, and let us estimate $r>0$ such that the solution of $(*)$ with initial condition $\left(0, y_{0}\right)$ can be extended to $D_{r}$, in such a way that its image is contained in $P$. Denote this solution by $(x(T), y(T))$. For a fixed $w \in \mathbb{C}$ with $|w|=1$, we have

(**) $\quad x(t . w)=\int_{0}^{t} p w(y(s . w))^{p-1} d s$ and $y(t . w)=y_{o}+\int_{0}^{t} q w(x(s . w))^{q-1} d s$.

Set $X(t)=|x(t . w)|$ and $Y(t)=|y(t . w)|$. It follows from $(* *)$ that

$$
(* * *) \quad X(t) \leq \int_{0}^{t} p(Y(s))^{p-1} d s \text { and } Y(t) \leq\left|y_{o}\right|+\int_{0}^{t} q(X(s))^{q-1} d s .
$$

Let $(Z(t), W(t))$ be the (real) solution of $(*)$ with initial condition $Z(0)=0, W(0)=$ $\left|y_{o}\right|$. It follows from Proposition 6 that $X(t) \leq Z(t)$ and $Y(t) \leq W(t)$ for $t \in[0, b)$, where $b>0$ is the maximal interval of definition of $(Z(t), W(t))$. In particular, $(x(t . w), y(t . w))$ can be extended to this interval. Now, since $y^{p}-x^{q}$ is a first integral of $(*)$, we must have $(W(t))^{p}-(Z(t))^{q}=(W(0))^{p}-(Z(0))^{q}=\left|y_{o}\right|^{p}$, which implies that $Z(t)=\left[(W(t))^{p}-\left|y_{o}\right|^{p}\right]^{1 / q} \leq(W(t))^{p / q}$. Therefore,

$$
W(t)=\left|y_{o}\right|+\int_{0}^{t} q(Z(s))^{q-1} d s \leq\left|y_{o}\right|+\int_{0}^{t} q(W(s))^{p(q-1) / q} d s .
$$

Let $A(t)$ be the solution of $\frac{d A}{d t}=q \cdot A^{p(q-1) / q}$ with $A(0)=W(0)=\left|y_{o}\right|$. Then $W(t) \leq A(t)$ (Proposition 6) and

$$
A(t)=\frac{\left|y_{o}\right|}{\left[1-\frac{q^{2}}{N}\left|y_{o}\right|^{N / q} \cdot t\right]^{q / N}},
$$

where $N=p q-p-q$. Now, suppose that $A(t)<1$ for $t$ in some interval $[0, c)$. It follows that $Z(t) \leq(W(t))^{p / q} \leq(A(t))^{p / q}<1$, so that $|x(t . w)| \leq Z(t)<1$ and $|y(t . w)| \leq W(t)<1$, which implies that $(x(t . w), y(t . w)) \in P$. On the other hand, the solution in $t$ of the inequality $A(t)<1$ is

$$
t<\frac{N}{q^{2}} \cdot \frac{1-\left|y_{o}\right|^{N / q}}{\left|y_{o}\right|^{N / q}}
$$

so that if $\left|y_{o}\right| \leq 1 / 2$, we get that $(x(t . w), y(t . w))$ can be extended to the interval $[0, r)$, where $r=\frac{c}{\left|y_{o}\right|^{N / q}} \leq \frac{N}{q^{2}} \frac{1-\left|y_{o}\right|^{N / q}}{\left|y_{o}\right|^{N / q}}$ and $c=\frac{N}{q^{2}}\left(1-(1 / 2)^{N / q}\right)$. This proves that the solution $(x(T), y(T))$ can be extended to the disk of radius $r$, in such a way that its image is contained in $P$. It follows from Lemma 3 that

$$
\lambda_{P}\left(0, y_{o}\right) \geq r^{2}\left|\tilde{X}\left(0, y_{o}\right)\right|^{2}=a \cdot \frac{\left|y_{o}\right|^{2(p-1)}}{\left|y_{o}\right|^{2 N / q}}=a \cdot\left|y_{o}\right|^{2 p / q} \text { if }\left|y_{o}\right|<1 / 2,
$$

where $a=p^{2} \cdot c^{2}$, which proves the assertion. 
Now the assertion implies that

$$
\left.\mu_{P}\right|_{(0, y)}=\frac{4|d z|^{2}}{\lambda_{P}(0, y)} \leq C \cdot \frac{|d z|^{2}}{|y|^{2 p / q}},
$$

where $C>0$ is some constant. Consider the curve $\gamma(t)=(0, t), t \in[0,1 / 2]$. If $\ell$ is its length in the metric $\mu_{P}$, then

$$
\ell=\int_{\gamma} \sqrt{\mu_{P}} \leq \sqrt{C} \int_{\gamma} \sqrt{\frac{|d z|^{2}}{|y|^{2 p / q}}}=\sqrt{C} \int_{0}^{1 / 2} \frac{d t}{t^{p / q}}<+\infty,
$$

because $p / q<1$. This implies that $\mu_{P}$ is not complete at 0 .

We observe that the same type of argument works for arbitrarily small polydisks $P_{\rho}=\{(x, y) ;|x|<\rho,|y|<\rho\}$. This implies that the germ of $\mathcal{F}$ at 0 does not satisfy property $\mathbf{P . 2}$ at 0 . We leave the details for the reader.

In fact, based in the above argument, we can state the following:

Proposition 8. Let $\mathcal{F}$ be a holomorphic foliation in a complex surface $M$, and let $g$ be a (continuous) hermitian metric on $M$. Suppose that all leaves of $\mathcal{F}$ are hyperbolic and that $\mathcal{F}$ has a singularity $p$ with the following property: there exists a holomorphic coordinate system $(\phi=(x, y), U)$ around $p$ such that $x(p)=y(p)=0$ and $G(x, y)=y^{p}-x^{q}$ is a first integral of $\left.\mathcal{F}\right|_{U}$, where $1<p<q$. Let $\lambda=\lambda_{\mathcal{F}, g}$ and $\mu=\frac{4 g}{\lambda}$. Then $\mu$ is not complete at $p$.

Proof. After composition of $\phi$ with a homothety of $\mathbb{C}^{2}$, we can suppose that $\phi(U) \supset$ $\bar{P}$, where $P=\{(x, y) ;|x|<1|y|<1\}$. Let $\lambda_{1}=\lambda_{\left.\mathcal{F}\right|_{P}, g}, \lambda_{P}=\lambda_{\left.\mathcal{F}\right|_{P},|d z|^{2}}, \mu_{1}=\frac{4 g}{\lambda_{1}}$ and $\mu_{P}=\frac{4|d z|^{2}}{\lambda_{2}}\left(|d z|^{2}=|d x|^{2}+|d y|^{2}\right)$. It follows from Proposition 4 that $\lambda_{1} \leq \lambda$, so that $\mu \leq \mu_{1}$. On the other hand, $\left.g\right|_{P}$ is equivalent to $|d z|^{2}$, in the sense that there exists a constant $K>1$ such that $K^{-1}|v|^{2} \leq g_{z}(v) \leq K|v|^{2}$ for any $z \in P$ and any $v \in T_{z} M$. This implies that $K^{-1} \lambda_{P}(z) \leq \lambda_{1}(z) \leq K \lambda_{P}(z)$ for all $z \in P$, so that

$$
K^{-2} \cdot \frac{4|v|^{2}}{\lambda_{P}(z)} \leq \frac{4 g_{z}(v)}{\lambda_{1}(z)} \leq K^{2} \cdot \frac{4|v|^{2}}{\lambda_{P}(z)}, \forall z \in P, \forall v \in T_{z} M
$$

Let $\gamma$ be the curve of the previous example. From the above inequalities we get

$$
\int_{\gamma} \sqrt{\mu} \leq \int_{\gamma} \sqrt{\mu_{1}} \leq K \cdot \int_{\gamma} \sqrt{\mu_{P}}<+\infty .
$$

Therefore $\mu$ is not complete at $p$.

§3.3. Proof of Theorem 1 . In this section $\mathcal{F}$ will be a germ of foliation at $0 \in \mathbb{C}^{2}$ with a saddle-node at 0 . This type of foliation can be defined by a germ of vector field $Y$ at $0 \in \mathbb{C}^{2}$ whose eigenvalues of $D Y(0)$ are $\lambda_{1}=0$ and $\lambda_{2} \neq 0$. The main reference for this type of singularity will be [M-R. It is well known that, after dividing $Y$ by a germ of function $u \in \mathcal{O}^{*}$, then, for any $k \geq p+1$, there exists a coordinate system $(x, y)$ around 0 , such that

$$
\frac{1}{u} . Y=X(x, y)=x^{p+1} \partial / \partial x+\left[y\left(1+\alpha x^{p}\right)+x R(x, y)\right] \partial / \partial y,
$$

where $R$ has multiplicity at least $k$ at $0 \in \mathbb{C}^{2}$. This form is known as "Dulac's normal form". As a consequence, it is possible to prove that $Y$ is formally equivalent to a vector field of the type $\hat{f}\left[x^{p+1} \partial / \partial x+y\left(1+\alpha x^{p}\right) \partial / \partial y\right]$, where $\hat{f}$ is a formal series. The vector field

$$
x^{p+1} \partial / \partial x+y\left(1+\alpha x^{p}\right) \partial / \partial y
$$


is known as the formal normal form of $\mathcal{F}$. We will prove that $\mathcal{F}$ satisfies property P.2, and therefore it also satisfies property P.1. Let us begin with the case in which $\mathcal{F}$ has two local separatrices through 0 . In fact we will prove a more general result.

Proposition 9. Let $\mathcal{F}$ be a germ of holomorphic foliation at $0 \in \mathbb{C}^{n}$ with an isolated singularity at 0 . Suppose that $\mathcal{F}$ has $n$ smooth hypersurfaces invariant, which meet in general position at 0 . Then $\mathcal{F}$ satisfies property $\mathbf{P . 2}$.

Proof. Consider a local coordinate system at $0 \in \mathbb{C}^{n}$, say $\left(z_{1}, \ldots, z_{n}\right)$, such that the invariant hypersurfaces are $\left(z_{j}=0\right), j=1, \ldots, n$. In this case, $\mathcal{F}$ is defined by a vector field $X$ as below:

$$
X(z)=z_{1}^{k_{1}} \cdot P_{1}(z) \partial / \partial z_{1}+\ldots+z_{n}^{k_{n}} \cdot P_{n}(z) \partial / \partial z_{n},
$$

where $P_{1}, \ldots, P_{n} \in \mathcal{O}_{n}$ and $k_{1}, \ldots, k_{n} \in \mathbb{N}$. Let us fix a ball $B_{r}$ such that $X$ has a representative defined in $B_{r}$, still denoted by $X$, such that 0 is the unique singularity of $X$ in $B_{r}$. After a homothety, if necessary, we can suppose that $B_{r}$ contains the polydisk $P=\left\{\left(z_{1}, \ldots, z_{n}\right) ;\left|z_{j}\right|<1, j=1, \ldots, n\right\}$. Let $\lambda=\lambda_{\mathcal{F}_{P}, g}$ and $\mu=\frac{4|d z|^{2}}{\lambda}$. Define $\psi_{j}: P \backslash\{0\} \rightarrow \mathbb{R}$ by

$$
\psi_{j}(z)=\frac{\left|z_{j}\right|^{2 k_{j}-2}\left|P_{j}(z)\right|^{2}}{\left(\ln \left(\left|z_{j}\right|\right)\right)^{2}} \text { if } z_{j} \neq 0 \text { and } \psi_{j}(z)=0 \text { if } z_{j}=0 .
$$

It is not difficult to see that $\psi_{j}$ is continuous in $P \backslash\{0\}$. Let $\psi(z)=\max \left\{\psi_{1}(z), \ldots\right.$, $\left.\psi_{n}(z)\right\}$. Since 0 is the unique singularity of $X$ in $B_{r}$, it follows that $\psi(z)>0$ for all $z \in P \backslash\{0\}$. Moreover $\psi$ is continuous. Consider the metric $g_{z}=\frac{\psi(z)}{|X(z)|^{2}} \cdot|d z|^{2}$.

Assertion 1. $g$ is $\mathcal{F}$-ultrahyperbolic with curvature bounded by -1. In particular, $g_{z} \leq \mu_{z}$ for all $z \in P$.

Proof. Fix $z_{o}=\left(z_{1}, \ldots, z_{n}\right) \in P \backslash\{0\}$ and $j \in\{1, \ldots, n\}$ such that $\psi(z)=\psi_{j}(z)$. Let $z(T)=\left(z_{1}(T), \ldots, z_{n}(T)\right)$ be the solution of $\frac{d z}{d T}=X(z)$ with initial condition $z(0)=z_{o}$, which can be defined in some disk $D_{\epsilon} \subset \mathbb{C}$. Then $z^{*}(g)=\psi(z(T))|d T|^{2} \geq$ $\psi_{j}(z(T))|d T|^{2}$. Now, observe that $\psi(z(0))=\psi_{j}(z(0))$ and

$\psi_{j}(z(T))|d T|^{2}=\frac{\left|z_{j}(T)\right|^{2 k_{j}}\left|P_{j}(z(T))\right|^{2}}{\left|z_{j}(T)\right|^{2} \cdot \ln ^{2}\left(\left|z_{j}(T)\right|\right)}=\frac{\left|\frac{d z_{j}}{d T}(T)\right|^{2}}{\left|z_{j}(T)\right|^{2} \cdot \ln ^{2}\left(\left|z_{j}(T)\right|\right)}=z_{j}^{*}\left[\frac{|d w|^{2}}{|w|^{2} \cdot \ln ^{2}(|w|)}\right]$.

Since $\frac{|d w|^{2}}{|w|^{2} \cdot \ln ^{2}(|w|)}$ is the Poincaré metric of $\mathbb{D}^{*}=\mathbb{D} \backslash\{0\}$ (cf. $[\underline{\mathrm{K}}]$ ), it follows that the gaussian curvature of $\psi_{j}(z(T))|d T|^{2}$ is -1 . Therefore $g$ is $\mathcal{F}$-ultrahyperbolic with curvature bounded by -1 . Now, $\mu$ induces the Poincaré metric on the leaves of $\left.\mathcal{F}\right|_{P}$. This fact and Ahlfors Lemma imply that for any $z \in P \backslash\{0\}$ and $v \in T_{z}\left(L_{z}\right)$, we have

$$
\mu_{z}(v)=\frac{4|v|^{2}}{\lambda(z)} \geq g_{z}(v)=\frac{\psi(z)}{|X(z)|^{2}} \cdot|v|^{2} \Longrightarrow \lambda(z) \leq \frac{|X(z)|^{2}}{4 \psi(z)} \Longrightarrow \mu_{z} \geq g_{z},
$$

which proves the assertion.

Assertion 2. $\mu_{z} \geq g_{z} \geq \frac{|d z|^{2}}{n|z|^{2} \cdot \ln ^{2}(|z|)}$ if $|z|<1 /$ e. In particular $\mu$ is complete at 0 and $\mathcal{F}$ satisfies property $\mathbf{P . 2}$. 
Proof. Fix $z=\left(z_{1}, \ldots, z_{n}\right)$ with $|z|^{2}=\sum_{i=1}^{n}\left|z_{i}\right|^{2}<1 / e^{2}$ and $j \in\{1, \ldots, n\}$ such that $\psi(z)=\psi_{j}(z)$. Since $\left|z_{i}\right|^{2} \cdot \ln ^{2}\left(\left|z_{i}\right|\right) \leq|z|^{2} \cdot \ln ^{2}(|z|)$ for all $i=1, \ldots, n$ such that $0<\left|z_{i}\right| \leq|z|<1 / e$, we have

$$
\psi(z)=\psi_{j}(z) \geq \psi_{i}(z)=\frac{\left|z_{i}\right|^{2 k_{i}}\left|P_{i}(z)\right|^{2}}{\left|z_{i}\right|^{2} \cdot \ln ^{2}\left(\left|z_{i}\right|\right)} \geq \frac{\left|z_{i}\right|^{2 k_{i}}\left|P_{i}(z)\right|^{2}}{|z|^{2} \cdot \ln ^{2}(|z|)},
$$

for all $i=1, \ldots, n$ such that $z_{i} \neq 0$. It follows that

$$
n . \psi(z) \geq \frac{1}{|z|^{2} \cdot \ln ^{2}(|z|)} \sum_{i=1}^{n}\left|z_{i}\right|^{2 k_{i}}\left|P_{i}(z)\right|^{2}=\frac{|X(z)|^{2}}{|z|^{2} \cdot \ln ^{2}(|z|)},
$$

which implies that

$$
g_{z}=\frac{\psi(z)}{|X(z)|^{2}} \cdot|d z|^{2} \geq \frac{|d z|^{2}}{n|z|^{2} \cdot \ln ^{2}(|z|)} \text { if }|z|<1 / e,
$$

so that $\mu_{z} \geq \frac{|d z|^{2}}{n|z|^{2} \cdot \ln ^{2}(|z|)}$, if $|z|<1 / e$. Since $\frac{|d z|^{2}}{|z|^{2} \cdot \ln ^{2}(|z|)}$ is complete at 0 , this proves Assertion 2 and the proposition.

Proposition 9 proves Theorem 1 in the case where the saddle-node has two local separatrices through $0 \in \mathbb{C}^{2}$. However, in general, a saddle-node has just one local separatrix through 0 . In this case, in order to construct an $\mathcal{F}$-ultrahyperbolic metric $g$ as in Proposition 9, we have to use the construction of Martinet-Ramis (cf. M-R]), which gives the analytic moduli in terms of the formal normal form. Next we describe the construction of Martinet-Ramis.

Let us consider a saddle-node given by a vector field

$$
X(x, y)=x^{p+1} \partial / \partial x+\left[y\left(1+\alpha x^{p}\right)+x R(x, y)\right] \partial / \partial y
$$

where $R$ has multiplicity at least $p+1$ at $0 \in \mathbb{C}^{2}$. The construction of MartinetRamis is based on a Theorem of Hukuara, Kimura and Matuda (cf. [H-K-M]).

Theorem. Let $S \subset \mathbb{C}$ be a sector of the form

$$
S=\left\{x=|x| e^{i \theta} ; 0<|x|<r \text { and } \theta_{o}<\theta<\theta_{1}\right\} \cup\{0\},
$$

where $r$ is small and $\theta_{1}-\theta_{o}<2 \pi / p$. Then there exists an analytic change of coordinates $\phi_{S}: S \times D \rightarrow S \times \mathbb{C}$, where $D$ is a disk with center $0 \in \mathbb{C}$, such that $\phi_{S}(x, y)=(x, \psi(x, y))$ and

$$
\left(\phi_{S}\right)_{*}(X)(x, y)=u(x, y) \cdot\left[x^{p+1} \partial / \partial x+y\left(1+\alpha x^{p}\right) \partial / \partial y\right]
$$

where $u(0,0) \neq 0$. Moreover, $\phi_{S}$ is asymptotic at 0 to the formal transformation which sends $X$ to its formal normal form.

In other words $X$ is equivalent to its formal normal form in $S \times D$ by a transformation of the type $\phi_{S}(x, y)=(x, \psi(x, y))$. We will call a transformation of this type a fibered transformation. We say that $\phi_{S}$ is a sectorial normalization of $X$.

Corollary. Let $S$ be as in the Theorem and $\phi_{S}^{1}, \phi_{S}^{2}$ be two sectorial normalizations of $X$. Then $\phi_{S}^{2} \circ\left(\phi_{S}^{1}\right)^{-1}$ sends the formal normal form to itself in $S \times D$ and is asymptotic to the identity along the axis $(x=0)$.

Observe that $\phi_{S}^{2} \circ\left(\phi_{S}^{1}\right)^{-1}$ is fibered, that is, $\phi_{S}^{2} \circ\left(\phi_{S}^{1}\right)^{-1}(x, y)=\left(x, g_{S}(x, y)\right)$. We call the transformation $g_{S}$, as above, a sectorial isotropy of $X$. We have the following theorem. 
Theorem ([M-R]). Let $S$ be a sector as above and let $g_{S}$ be a sectorial isotropy. Let $\Phi(x)=x^{\alpha} \cdot \exp \left(-1 / p x^{p}\right)$ be a univalent branch of solution of $\frac{d y}{d x}=\frac{y\left(1+\alpha x^{p}\right)}{x^{p+1}}$ defined in $S$. We have:

(a) If $S \subset\left\{x \in \mathbb{C}\right.$; $\left.\operatorname{Re}\left(x^{p}\right)>0\right\}$, then $g_{S}(x, y)=y+c . \Phi(x)$, where $c \in \mathbb{C}$.

(b) If $S \subset\left\{x \in \mathbb{C} ; \operatorname{Re}\left(x^{p}\right)<0\right\}$, then there exists a convergent series $h(y)=$ $y+\sum_{n=2}^{\infty} a_{n} y^{n}=y \cdot u(y)$ such that $g_{S}(x, y)=y \cdot u(y / \Phi(x))$.

In case (a), that is, $S \subset\left\{x \in \mathbb{C} ; \operatorname{Re}\left(x^{p}\right)>0\right\}$, we say that $S$ is an attracting sector, whereas in case (b) we say that $S$ is a saddle sector. Observe that, in any case, $g_{S}$ sends solutions of the linear equation $\frac{d y}{d x}=\frac{y\left(1+\alpha x^{p}\right)}{x^{p+1}}$ on $S$ onto solutions of the same equation. The space of such solutions is $\mathbb{C}$, in the case of an attracting sector, and a disk $D$ centered at $0 \in \mathbb{C}$ in the case of a saddle sector. In the first case, $g_{S}$ acts as a translation in the space of solutions, whereas $g_{S}$ acts as $h(y)$ in the second case. We will denote by $\mathcal{H}$ the set of convergent series $h(y)=y+\sum_{n=2}^{\infty} a_{n} y^{n}$.

Now consider a covering of $D_{r}=\{x ;|x|<r\}$ by $2 p$ sectors $S_{1}, \ldots, S_{2 p}$ :

$$
S_{j}=S_{j}(r)=\left\{x=|x| e^{i \theta} ;|x|<r \text { and } \theta_{j}-a<\theta<\theta_{j}+a\right\},
$$

where $\pi / 2 p<a<\pi / p$ and $\theta_{j}=\frac{2 j-1}{2 p} \pi$, so that $\cos \left(p \theta_{j}\right)=0, j=1, \ldots, 2 p$. Set

$$
A_{j}=A_{j}(r)=\left\{x=|x| e^{i \theta} ;|x|<r \text { and } \frac{j \pi}{p}-b<\theta<\frac{j \pi}{p}+b\right\},
$$

where $b=a-\frac{\pi}{2 p}$, so that $A_{j}=S_{j} \cap S_{j+1}\left(S_{2 p+1}=S_{1}\right)$ if $p \geq 2$, and $S_{1} \cap S_{2}=A_{1} \cup A_{2}$ if $p=1$. Observe that $\operatorname{Re}\left(x^{p}\right)>0$ if $x \in A_{j}$ and $j$ is even, and $\operatorname{Re}\left(x^{p}\right)<0$ if $x \in A_{j}$ and $j$ is odd. It follows that $A_{j}$ is a saddle sector if $j$ is even and an attracting sector if $j$ is odd. Therefore, for $j$ even the isotropy $g_{A_{j}}$ acts in the space of leaves as an element $h_{j} \in \mathcal{H}$, and for $j$ odd $g_{A_{j}}$ acts as a translation $h_{j}(y)=y+c_{j}$. Therefore we can define a map $\mathcal{M}$ from the space of germs of vector fields $X$ as above into $\mathbb{C}^{p} \times \mathcal{H}^{p}$ by $\mathcal{M}(X)=\left(c_{1}, \ldots, c_{2 p-1}, h_{2}, \ldots, h_{2 p}\right)$.

Now we can state the classification Theorem of Martinet-Ramis.

Theorem $([\underline{M-R}])$. The map $\mathcal{M}$ is a bijection.

Another way of stating the above result is that any saddle-node $X$ with a fixed formal normal form, say $x^{p+1} \partial / \partial x+y\left(1+\alpha x^{p}\right) \partial / \partial y$, can be obtained by glueing the foliations defined by the normal form in the sectors $S_{j}$ and $S_{j+1}$, in $A_{j}$ by using the the sectorial isotropy $g_{A_{j}}$. In particular, we get the following result:

Corollary $([\mathrm{M}-\mathrm{R}]) . X$ has two local analytic separatrices through 0 if, and only if, $\mathcal{M}(X)=\left(0, \ldots, 0, h_{2}, \ldots, h_{2 p}\right)$, that is, all translations vanish.

Let us now prove that the metric $\frac{4|d z|^{2}}{\lambda_{P}(z)}$ is complete, in the case that $X$ has just one local analytic separatrix through 0 . Let $S_{j}(r)$ and $A_{j}(r), j=1, \ldots, 2 p$, be as before. Consider coordinates $\left(x, Y_{j}\right)$ in $S_{j}(r)$, and the sectorial normalizations $\phi_{j}(x, y)=\left(x, Y_{j}(x, y)\right)=\left(x, Y_{j}\right), j=1, \ldots, 2 p$, which send $X$ to the formal normal form. Observe that, since we can suppose that $X$ is tangent to the formal normal form up to any order, we can suppose that $\phi_{j}$ is asymptotic to the identity in order $k \geq 2$ in the sector $S_{j}(r)$, that is,

$$
\lim _{x \in S_{j}(r), x \rightarrow 0} \frac{\left|Y_{j}(x, y)-y\right|}{|x|^{k}}=0 .
$$


Define in $S_{j}(r) \times D, j=1, \ldots, 2 p, r<1$, the following metrics:

$$
h_{j,\left(x, Y_{j}\right)}=\max \left\{\frac{|x|^{2}}{\ln ^{2}(|x|)}, \psi\left(x, Y_{j}\right)\right\} \cdot \frac{|d x|^{2}+\left|d Y_{j}\right|^{2}}{|x|^{4}+|1+\alpha x|^{2}\left|Y_{j}\right|^{2}},
$$

where $\psi(x, Y)=\frac{|1+\alpha x|^{2}}{(\ln (|Y|))^{2}}$ if $Y \neq 0$ and $\psi(x, Y)=0$ if $Y=0$. It is not difficult to see that $h_{j}$ is continuous for $j=1, \ldots, 2 p$. Moreover, it follows from Assertion 2 in the proof of Proposition 9 that

$$
h_{j,\left(x, Y_{j}\right)} \geq \frac{|d x|^{2}+\left|d Y_{j}\right|^{2}}{\left|Z_{j}\right|^{2} \cdot \ln ^{2}\left(\left|Z_{j}\right|\right)}, \text { if }\left|Z_{j}\right|<1 / e,
$$

where $Z_{j}=\left(x, Y_{j}\right)$. Since $F_{j}$ is asymptotic to the identity in order 2 when $x \rightarrow 0$, it follows that there are constants $C>1$ and $\rho>0$ such that

$$
C^{-1} \frac{|d z|^{2}}{|z|^{2} \ln ^{2}(|z|)} \leq \phi_{j}^{*}\left(h_{j}\right)_{z} \leq C \frac{|d z|^{2}}{|z|^{2} \ln ^{2}(|z|)},
$$

where $z=(x, y),|z|<\rho$. To complete the proof, it is enough to prove there exist a polydisk $P=\{(x, y) ;|x|<r,|y|<r\}$ such that if $\lambda=\lambda_{P}$, then

$$
\frac{4|d z|^{2}}{\lambda(z)} \geq \phi_{j}^{*}\left(h_{j}\right)_{z} \text { for } z \in P_{j}=P \cap\left(S_{j}(r) \times \mathbb{C}\right) .
$$

We will prove this fact in the case $p=1$ and leave the proof of the general case for the reader. In this case, since $p=1$ and $X$ has just one local analytic separatrix through 0 , we have $\mathcal{M}(X)=\left(c_{o}, h\right) \in \mathbb{C} \times \mathcal{H}$, where $c_{o} \neq 0$. Therefore, the glueing maps $F_{j}: A_{j}(r) \times D \rightarrow A_{j}(r) \times \mathbb{C}$ can be written as

$$
F_{1}\left(x, Y_{1}\right)=\left(x, Y_{1}+c_{o} \Phi(x)\right)=\left(x, Y_{2}^{1}\left(x, Y_{1}\right)\right) \text { if }\left(x, Y_{1}\right) \in A_{1}(r) \times \mathbb{C}
$$

and

$$
F_{2}\left(x, Y_{1}\right)=\left(x, Y_{1} \cdot u\left(Y_{1} / \Phi(x)\right)\right)=\left(x, Y_{2}^{2}\left(x, Y_{1}\right)\right) \text { if }\left(x, Y_{1}\right) \in A_{2}(r) \times D,
$$

where $h(y)=y \cdot u(y)$ and $\Phi(x)=x^{\alpha} \cdot \exp (-1 / x)$. Observe that

$$
\lim _{x \in A_{j}(r), x \rightarrow 0} \frac{\left|Y_{2}^{j}\left(x, Y_{1}\right)-Y_{1}\right|}{|x|^{m}}=0,
$$

for $j=1,2$ and $m \geq 1$, so that $F_{j}$ is asymptotic to the identity on $A_{j}(r) \times D$. Denote by $\mathcal{F}$ the foliation defined by $X$.

Assertion 3. There exists $r>0$ with the following property:

(P) Let $P=\{(x, y) ;|x|<r,|y|<r\}$ and $P_{j}=P \cap\left(S_{j}(r) \times D_{r}\right)$. Given $z_{o}=\left(x_{o}, y_{o}\right) \in P_{j}, q_{o}=\phi_{j}\left(z_{o}\right)$, if $L_{z_{o}}^{j}$ is the connected component of $L_{z_{o}} \cap P_{j}$ which contains $z_{o}$, then the function

$$
\left.\pi_{2} \circ \phi_{j}\right|_{L_{z_{o}}^{j}}: L_{z_{o}}^{j} \rightarrow \mathbb{C}
$$

has an analytic extension to $L_{z_{o}}$, where $L_{z_{o}}$ denotes the leaf of $\left.\mathcal{F}\right|_{P}$ through $z_{o}$ and $\pi_{2}$ the $\operatorname{map} \pi_{2}\left(x, Y_{j}\right)=Y_{j}$.

Let us assume Assertion 3 and prove $(*)$ from it. We have seen in Assertion 1 of the proof of Proposition 9 that the metric $h_{j}$ is $\mathcal{F}$-ultrahyperbolic of curvature bounded by -1 . This implies that $\phi_{j}^{*}\left(h_{j}\right)$ is also $\mathcal{F}$-ultrahyperbolic of curvature bounded by -1 . Lemma 4 implies that, for any $z_{o} \in P_{j}$, the metric $\left.\phi_{j}^{*}\left(h_{j}\right)\right|_{L_{z_{o}}^{j}}$ extends to a metric $g_{z_{o}}$ on $L_{z_{o}}$, the whole leaf of $\left.\mathcal{F}\right|_{P}$ through $z_{o}$. This metric is 
$\mathcal{F}$-ultrahyperbolic of curvature bounded by -1 . It follows from Ahlfors Lemma that

which implies

$$
\left.\frac{4|d z|^{2}}{\lambda}\right|_{L_{z_{o}}} \geq g_{z_{o}}
$$

$\left.\frac{4|d z|^{2}}{\lambda(z)}\right|_{L_{z_{O}}^{j}} \geq\left.\phi_{j}^{*}\left(h_{j}\right)\right|_{L_{z_{O}}^{j}} \geq\left. C^{-1} \frac{|d z|^{2}}{|z|^{2} \ln ^{2}(|z|)}\right|_{L_{z_{O}}^{j}} \Longrightarrow \lambda(z) \leq 4 C|z|^{2} \ln ^{2}(|z|), \forall z ! \in P_{j}$

so that

$$
\frac{4|d z|^{2}}{\lambda(z)} \geq C^{-1} \frac{|d z|^{2}}{|z|^{2} \ln ^{2}(|z|)}, \forall z \in P
$$

which concludes the proof of Theorem 1.

Proof of Assertion 3. Let us first consider the foliation $\mathcal{F}_{N}$, obtained by glueing the formal normal form by using the maps $F_{1}$ and $F_{2}$. Fix branchs of solutions $\Phi_{j}(x)=x^{\alpha} \cdot \exp (-1 / x), j=1,2$, of

$$
\frac{d Y}{d x}=\frac{Y(1+\alpha x)}{x^{p+1}}
$$

in the sectors $S_{j}(r)$. Any other solution of $(* *)$ in $S_{j}(r)$ can be written as $c . \Phi_{j}(x)$, $c \in \mathbb{C}$, and this solution gives origin to a leaf of $\mathcal{F}_{N}$ in the set $U_{j}(r, s)=s_{j}(r) \times$ $D_{s}$, which we denote by $L_{j}(r, s, c)$. It is not difficult to see that $L_{j}(r, s, c)$ is the intersection of the graph of $c . \Phi_{j}(x)$ with $U_{j}(r, s)$. Let us denote by $D_{j}(r, s, c)$ the set $\pi_{1}\left(L_{j}(r, s, c)\right)$, where $\pi_{1}$ is the first projection. Observe that

$$
L_{j}(r, s, c)=\left\{\left(x, c . \Phi_{j}(x)\right) ; x \in D_{j}(r, s, c)\right\}
$$

and

$$
D_{j}(r, s, c)=\left\{x \in S_{j}(r) ; c . \Phi_{j}(x) \in D_{s}\right\}=\left\{x \in S_{j}(r) ;\left|\Phi_{j}(x)\right|<\frac{s}{|c|}\right\}, \text { if } c \neq 0 .
$$

This implies the following facts:

(i) If $\left|c_{2}\right| \geq\left|c_{1}\right|>0$, then $D_{j}\left(r, s, c_{2}\right) \subset D_{j}\left(r, s, c_{1}\right)$.

(ii) If $s_{2} \geq s_{1}$, then $D_{j}\left(r, s_{1}, c\right) \subset D_{j}\left(r, s_{2}, c\right)$.

(iii) $D_{j}(r, s, 0)=S_{j}(r)$.

(iv) $D_{j}(r, s, c)$ is never empty. This fact follows from $\lim _{x \in A, x \rightarrow 0}\left|\Phi_{j}(x)\right|=0$, if $A$ is an attracting sector.

(v) There exists $M=M(r, s)>0$ such that if $|c|>M$, then

$$
L_{j}(r, s, c) \cap\left(A_{2}(r) \times D_{s}\right)=\emptyset .
$$

This follows from the fact that $\lim _{x \in A_{2}(r), x \rightarrow 0}\left|\Phi_{j}(x)\right|=+\infty\left(A_{2}(r)\right.$ is a saddle sector).

It follows from (v) that if $|c|>M(r, s)$, then the leaf $L_{j}(r, s, c)$ "ends" somewhere between the extreme directions of $S_{j}(r)$. Observe also that

(vi) $\lim _{r \rightarrow 0} M(r, s)=0$.

Now fix a leaf $L_{1}(r, s, c)$ in $U_{1}(r, s)$ and let us see how this leaf continues in $U_{2}(r, s)$, after the identifications $F_{1}$ and $F_{2}$. Since $\Phi_{1}$ and $\Phi_{2}$ are solutions of (**) in the sectors $A_{1}(r)$ and $A_{2}(r)$, we have that $\Phi_{2}(x) / \Phi_{1}(x)$ are constants in these sectors, say $\Phi_{2}(x) / \Phi_{1}(x)=c_{j} \neq 0$ in $A_{j}(r), j=1,2$.

We have

$$
F_{1}\left(x, c . \Phi_{1}(x)\right)=\left(x,\left(c+c_{o}\right) \Phi_{1}(x)\right)=\left(x, \frac{c+c_{o}}{c_{1}} \Phi_{2}(x)\right),
$$


so that $L_{1}(r, s, c)$ continues as $L_{2}\left(r, s, T_{1}(c)\right)$, after the identification $F_{1}$, where $T_{1}(c)=\frac{c+c_{o}}{c_{1}}$. With the same type of argument, it is possible to prove $L_{1}(r, s, c)$ continues as $L_{2}\left(r, s, T_{2}(c)\right)$, after the identification $F_{2}$, where $T_{2}(c)=\frac{1}{c_{2}} h(c)$. Therefore the leaf $L_{1}(r, s, c)$ continues in the negative diretion of the angle according with the following scheme:

$$
\begin{aligned}
L_{1}(r, s, c) & \rightarrow L_{2}\left(r, s, T_{1}(c)\right) \rightarrow L_{1}\left(r, s, T_{2}^{-1} \circ T_{1}(c)\right) \\
& \rightarrow L_{2}\left(r, s, T_{1} \circ T_{2}^{-1} \circ T_{1}(c)\right) \rightarrow \ldots
\end{aligned}
$$

and in the positive direction of the angle as

$$
\begin{aligned}
L_{1}(r, s, c) & \rightarrow L_{2}\left(r, s, T_{2}(c)\right) \rightarrow L_{1}\left(r, s, T_{1}^{-1} \circ T_{2}(c)\right) \\
& \rightarrow L_{2}\left(r, s, T_{2} \circ T_{1}^{-1} \circ T_{2}(c)\right) \rightarrow \ldots
\end{aligned}
$$

Since $T_{1}(c)=c / c_{1}+k_{1}$ and $T_{1}^{-1}(c)=c_{1} \cdot c+k_{2}$, where $k_{1}=c_{o} / c_{1} \neq 0$ and $k_{2}=-c_{o}$, it follows from (vi) that if $r$ and $s$ are small, then

$$
\inf \left\{\left|T_{1}(c)\right|,\left|T_{1}^{-1}(c)\right| ; c \in D_{s}\right\}>M(r, s) .
$$

In this case, we get from ( $\mathbf{v})$ that all leaves $L_{1}(r, s, c)$ have at most one continuation in the negative direction and two continuations in the positive direction. Similarly, if $r$ and $s$ are small, then any leaf $L_{2}(r, s, c)$ has at most one continuation in the positive direction and two in the negative direction.

Now fix $r_{o}, s_{o}>0$ as above and let $r$ be so small that $\phi_{j}\left(P_{j}\right) \subset\left(S_{j}\left(r_{o}\right) \times D_{s_{o}}\right)$. Denote by $\mathcal{F}_{N}$ the foliation obtained by the process of glueing described before and by $\mathcal{F}$ the foliation in $P$ defined by $X$. Fix $z_{o} \in P$, say $z_{o} \in P_{1}$ (for $z_{o} \in P_{2}$ the argument is similar) and let $L$ be the leaf of $\mathcal{F}$ through $z_{o}$ and $L_{1}$ be the connected component of $L \cap P_{1}$ which contains $z_{o}$. If $Z_{o}=\phi_{1}\left(z_{o}\right)$, then $Z_{o}$ belongs to some leaf $L_{1}(c):=L_{1}\left(r_{o}, s_{o}, c\right)$ of $\left.\mathcal{F}_{N}\right|_{U_{1}}$. It is clear that $\phi_{1}\left(L_{1}\right) \subset L_{1}(c)$. The leaf $L_{1}(c)$ is parametrized by

$$
x \in D_{1}(c) \mapsto\left(x, c . \Phi_{1}(x)\right),
$$

where $D_{1}(c):=D_{1}\left(r_{o}, s_{o}, c\right)$. This implies that, in this parametrization, we have

$$
\left.\pi_{2} \circ \phi_{1}\right|_{L_{1}}(x)=c . \Phi_{1}(x) .
$$

On the other hand, $L_{1}(c)$ continues in the negative direction as $L_{2}\left(T_{1}(c)\right):=$ $L_{2}\left(r_{o}, s_{o}, T_{1}(c)\right)$, and, in this direction, it ends in this step. This implies that, if $L_{1}$ continues in the negative direction (in $P$ ), then it has at most one continuation in this direction, say $L_{2}^{-} \subset P_{2}$, where $\phi_{2}\left(L_{2}^{-}\right) \subset L_{2}\left(T_{1}(c)\right)$. Since $L_{2}\left(T_{1}(c)\right)$ is parametrized by

$$
x \in D_{2}\left(T_{1}(c)\right) \mapsto\left(x, T_{1}(c) . \Phi_{2}(x)\right),
$$

it follows that, in this parametrization, $\left.\pi_{2} \circ \phi_{1}\right|_{L_{1}}$ can be continued analytically as $T_{1}(c) . \Phi_{2}(x)$. Similarly, in the positive direction, $L_{1}(c)$ and $L_{1}$ can be continued at most twice, the first as $L_{2}\left(T_{2}(c)\right)$ and $L_{1}\left(T_{1}^{-1} \circ T_{2}(c)\right)$, and the second, say as $L_{2}^{+}$and $L_{1}^{+}$, where $\phi_{2}\left(L_{2}^{+}\right) \subset L_{2}\left(T_{2}(c)\right)$ and $\phi_{1}\left(L_{1}^{+}\right) \subset L_{1}\left(T_{1}^{-1} \circ T_{2}(c)\right)$. Therefore $\left.\pi_{2} \circ \Phi_{1}\right|_{L_{1}}$ can be continued analytically in the positive direction as $T_{2}(c) . \Phi_{2}(x)$ in $L_{2}^{+}$and as $T_{1}^{-1} \circ T_{2}(c) \cdot \Phi_{1}(x)$ in $L_{1}^{+}$. Since (at most) four pieces cover the leaf $L$, this proves the assertion.

This finishes the proof of Theorem 1 . 


\section{REFERENCES}

[Ah-1] L. V. Ahlfors: "Conformal Invariants. Topics in Geometric Function Theory"; McGrawHill (1973). MR 50:10211

[Ah-2] L. V. Ahlfors: "An Extension of Schwarz' Lemma”; Trans. Am. Math. Soc., 43 (1938), pp. 359-364.

[C] A. Candel: "Uniformization of Surface Laminations"; Ann. Scient. de l'École Norm. Sup., 26(4) (1993), 489-515. MR 94f:57025

[C-G] A. Candel and X. Gomez-Mont: "Uniformization of the leaves of a Rational Vector Field"; Ann. de l'Institut Fourier 45, 4 (1995) 1123-1133. MR 96k:32068

[C-LN] C. Camacho and A. Lins Neto: "Geometric Theory of Foliations"; Birkhäuser, 1985. MR 87a:57029

[C-S] C. Camacho and P. Sad: "Invariant varieties through singularities of holomorphic vector fields"; Ann. of Math., 115 (1982). MR 83m:58062

[El1] E. L. Lima: "Grupo Fundamental e espaços de recobrimento"; Projeto Euclides, 1993.

[F-K] H. M. Farkas and I. Kra: "Riemann Surfaces"; Springer-Verlag, NY, 1980. MR 82c:30067]

$[\mathrm{H}-\mathrm{K}-\mathrm{M}]$ M. Hukuara, T. Kimura, and T. Matuda: "Équations differentielles ordinaires du premier ordre dans le champ complexe"; Publ. Math. Soc. of Japan (1961). MR 23:A1861

$[\mathrm{K}] \quad$ S. Kobayashi: "Hyperbolic manifolds and holomorphic maps"; Ed. Marcel Dekker (1970). MR 43:3503

[LN] A. Lins Neto: "Simultaneous Uniformization for the Leaves of Projective Foliations by Curves"; Boletim da Sociedade Brasileira de Matemática, vol. 25(2) (1994), pp. 181-206. MR 95k:32034

[M-P] W. Mello and J. Palis: "Geometric theory of dynamical systems"; Springer-Verlag (1982). MR 84a:58004

[M-R] J. Martinet and J. P. Ramis: "Problèmes des modules pour des équations différentielles non linéaires du premier ordre"; Publ. Math. de l'IHES 55 (1982). MR 84k:34011

[V] A. Verjovsky: "An Uniformization theorem for Holomorphic Foliations"; Contemp. Math. 58(III) (1987), pp. 233-253. MR 88h:57027

[P] O. Perron: "Eine neue Behandlung der ersten Randwertaufgabe für $\Delta u=0$ "; Math. Z. 18 (1923), 42-54. FM 49, 340.

Instituto de Matemática Pura e Aplicada, Estrada Dona Castorina, 110, Horto, Rio DE JANEIRO, BRASIL

E-mail address: alcides@impa.br

LCMAT-UEnF, Campos, Rio de Janeiro, Brasil

E-mail address: canille@uenf.br 